This item was submitted to Loughborough's Research Repository by the author.

Items in Figshare are protected by copyright, with all rights reserved, unless otherwise indicated.

\title{
Backpropagating constraints-based trajectory tracking control of a quadrotor with constrained actuator dynamics and complex unknowns
}

\section{PLEASE CITE THE PUBLISHED VERSION}

https://doi.org/10.1109/TSMC.2018.2834515

PUBLISHER

(C) IEEE

VERSION

AM (Accepted Manuscript)

\section{LICENCE}

CC BY-NC-ND 4.0

\section{REPOSITORY RECORD}

Wang, Ning, Shun-Feng Su, Min Han, and Wen-Hua Chen. 2018. "Backpropagating Constraints-based Trajectory Tracking Control of a Quadrotor with Constrained Actuator Dynamics and Complex Unknowns". Loughborough University. https://hdl.handle.net/2134/35022. 


\title{
Backpropagating Constraints Based Trajectory Tracking Control of a Quadrotor with Constrained Actuator Dynamics and Complex Unknowns
}

\author{
Ning Wang, Senior Member, IEEE, Shun-Feng Su, Fellow, IEEE, Min Han, Senior Member, IEEE, \\ and Wen-Hua Chen, Fellow, IEEE
}

\begin{abstract}
In this paper, a backpropagating constraints based trajectory tracking control (BCTTC) scheme is addressed for trajectory tracking of a quadrotor with complex unknowns and cascade constraints arising from constrained actuator dynamics including saturations and dead zones. The entire quadrotor system including actuator dynamics is decomposed into 5 cascade subsystems connected by intermediate saturated nonlinearities. By virtue of the cascade structure, backpropagating constraints (BC) on intermediate signals are derived from constrained actuator dynamics suffering from nonreversible rotations and nonnegative squares of rotors, and decouple subsystems with saturated connections. Combining with sliding-mode errors, BCbased virtual controls are individually designed by addressing underactuation and cascade constraints. In order to remove smoothness requirements on intermediate controls, first-order filters are employed, and thereby contributing to backsteppinglike sub-controllers synthesizing in a recursive manner. Moreover, universal adaptive compensators are exclusively devised to dominate intermediate tracking residuals and complex unknowns. Eventually, the closed-loop BCTTC system stability can be ensured by the Lyapunov synthesis, and trajectory tracking errors can be made arbitrarily small. Simulation studies demonstrate the effectiveness and superiority of the proposed BCTTC scheme for a quadrotor with complex constrains and unknowns.
\end{abstract}

Index Terms-Backpropagating constraints, quadrotor, constrained actuator dynamics, cascade constraints, complex unknowns, dead zones, trajectory tracking control.

\section{INTRODUCTION}

$\mathbf{R}$ ECENTLY, increasing attention has been paid to Vertical Take-Off and Landing (VTOL) unmanned aerial vehicles (UAV) pertaining to a wide area of vital applications including patrolling for forest fires, traffic monitoring and surveillance

Manuscript received December 30, 2016; revised July 23, 2017; January 21, 2018 and March 13; accepted April 30, 2018. This work is supported by the National Natural Science Foundation of P. R. China (under Grants 51009017 and 51379002), the Fund for Dalian Distinguished Young Scholars (under Grant 2016RJ10), the Innovation Support Plan for Dalian High-level Talents (under Grant 2015R065), and the Fundamental Research Funds for the Central Universities (under Grant 3132016314 and 3132018126).

N. Wang is with the Center for Intelligent Marine Vehicles, and the School of Marine Electrical Engineering, Dalian Maritime University, Dalian 116026 , China. email: n.wang.dmu.cn@gmail.com;

S.-F. Su is with the Department of Electrical Engineering, National Taiwan University of Science and Technology, Taiwan, P. R. China. email: sfsu@mail.ntust.edu.tw;

M. Han is with the School of Control Science and Engineering, Dalian University of Technology, Dalian 116023, P. R. China. email: minhan@dlut.edu.cn;

W.-H. Chen is with the Department of Aeronautical and Automotive Engineering, Loughborough University, Loughborough, LE113TU, UK email: w.chen@lboro.ac.uk rescue via hovering, tracking and coordination [1]-[4]. Recently, flapping-wing flying robotics have also attracted much attention by devising novel neuro-adaptive methods [5], [6]. Compared with fixed-wing aircrafts, the rotary-wing UAV possesses the significant advantage that it can take-off and land vertically in limited spaces and is easy to hover over the target. Note that the quadrotor UAV (QUAV) is a typical VTOL-UAV with simple mechanical structure and favorable maneuverability. In this context, as a remarkable platform of the UAV, the QUAV has attracted numerous research [7]-[10].

The QUAV is a highly nonlinear system with underactuated constraints and strong couplings between actuator dynamics, and thereby leading to great challenges in controller design and synthesis. With the development of advanced control approaches including sliding mode control (SMC) [11]-[13], dynamic surface control (DSC) [14], fuzzy/neural control [15]-[23], and non-smooth approaches [24]-[26] etc., promising control schemes for the QUAV are pursued ceaselessly. In the literature, control methods of the QUAV can be actually classified into two kinds, i.e., model-based approaches including feedback linearization [27] backstepping [28], SMC [29], adaptive control [30], model predictive control (MPC) [31], and robust control [32] etc., and mode-free approaches including PID [33]-[35], neural control [36] and fuzzy control [37], etc. Undoubtedly, the plant dynamics controlled would be dramatically simplified for linear/nonlinear PID controller design [38]. Backstepping- and SMC-based adaptive robust control schemes can incorporate complex unknown dynamics and even uncertainties and/or disturbances by employing disturbance observers [39], [40]. Furthermore, combining with modelfree approaches, i.e. adaptive fuzzy/neural approximators, for uncertainties and/or unknown dynamics, tracking errors of an uncertain QUAV can be made bounded [36], [41].

In order to facilitate trajectory/position tracking control of a QUAV, the entire QUAV system is usually decomposed into a cascaded form such that the underactuation issue can be solved by applying an inversion calculation to interconnected nonlinearities. To this end, various cascaded frameworks have been derived from QUAV kinematics and dynamics, and can be mainly classified into 3 categories, i.e., translational and rotational (TR) dynamics [42]-[45], underactuated and fullyactuated (UF) dynamics [46]-[49], and multiple-loop (ML) dynamics [50], [51]. Main ideas can be summarized as follows. Within the TR form, the orientation (attitude) variables are treated as virtual control inputs of translational dynamics in 
addition to actual thrusts generated by propellers. Furthermore, the desired attitude variables resulting from virtual control signals are taken as references to be tracked by rotational dynamics. In this context, virtual control inputs have to be reasonable since the total thrust is uniformly nonnegative. In terms of unit-quaternion, Abdessameud and Tayebi [3] created a tool for extracting the thrust and desired attitudes, whereby feasible magnitudes for intermediate signals can be ensured. Within the UF framework, underactuated dynamics are driven by one or more cascaded fully-actuated subsystems, and thereby rendering backstepping-based approaches available. Typically, inspired by Lyapunov's direct method for underactuated ship tracking [52], Do et al. [46] developed a global tracking control scheme for a QUAV by employing bounded backstepping techniques. Within the ML structure, several quasi-cascade loops are designed by employing timescale separation philosophy, whereby the innermost (outermost) loop has to possess the fastest (lowest) tracking error dynamics since virtual controllers can only stabilize individual subsystems. In summary, there exist the issues which are open:

- Dealing with cascade constraints. Using TR and UF cascade structures would inevitably ignore cascade constraints hidden within subsystems due to main facts as follows: (1) The total thrust is constrained by nonreversibly limited propeller rotations and has to be nonnegative; (2) Together with trigonometric functions of attitudes, desired cascade inputs to translational dynamics have to be feasibly constrained; (3) Cascade inputs to attitude dynamics are directly constrained by the squares of propeller rotations; (4) Thrust torques generated by individual propellers are restricted to be nonnegative and are determined by the squares of motor rotor speeds. (5) Moreover, DC actuators would also suffer from control input nonlinearities including saturations and dead zones. Note that an extraction algorithm for thrust and quaternion-based attitude has been addressed in [3]. However, it is limited to be available for quaternion-based models in addition that cascade constraints from rotation squares and actuator dynamics are still unsolved. Within the ML framework, interconnected nonlinearities between cascade dynamics can be characterized in a triangular-like form. Unfortunately, aforementioned cascade constraints have not been addressed to date [51], although individual loops can facilitate the SMC approach.

- Dealing with actuator dynamics. As analyzed above, actuator dynamics including transient responses and control input constraints would directly affect and limit the torque inputs to propeller rotation dynamics. Clearly, treating actuator dynamics as input nonlinearities/uncertainties [53], [54], linearized dynamics [43], [55], [56] or stationary mappings [1], [57] would hardly determine feasible input torques generated by propellers, and thereby resulting in uniformly unreachable regions within the desired control efforts. Nevertheless, BLDC motors in a QUAV are not allowed to rotate reversely such that uniformly upward thrust forces can be generated. In this context, it becomes empirical and risky to design control laws for torque inputs if BLDC dynamics are omitted and torque control signals are directly fed into the electronic speed control (ESC) module which generates 3-phase AC voltages via PWM signals. Hence, incorporating actuator dynamics into the QUAV model is strongly desirable for pursuing high autonomy. However, to our best knowledge, few attention to systematically dealing with actuator dynamics including control constraints has been paid for a QUAV.

In this paper, we focus on trajectory tracking control of a QUAV including cascade constraints, constrained actuator dynamics and complex unknowns, which is unsolved in the literature. By incorporating the SMC and DSC approaches into a backstepping-like framework, a backpropagating constraints (BC) based trajectory tracking control (BCTTC) scheme is proposed by devising extraction tools for cascade constraints. In the presence of actuator dynamics, unmodeled dynamics, uncertainties, measurement noises and external disturbances, the entire QUAV dynamics are formulated in a vectorial pure-feedback form with unmatched unknowns whereby intermediate constraints and underactuated dynamics appear in a cascade mode, and make traditional backstepping-based approaches unavailable. In this context, the BCTTC framework using the SMC is realized to circumvent both cascade constraints and underactuation issues, and recursively stabilize tracking errors. The DSC technique is further deployed to facilitate the derivation of intermediate signals. Since constrained actuator dynamics are sufficiently addressed, virtual control signals pertaining to Euler angles, rotation squares, and armature voltages of nonreversible motors with input saturations and dead zones are reasonably constrained by the $\mathrm{BC}$ extraction. In addition, intermediate tracking discrepancies and complex unknowns are further be attenuated by a family of universal adaptive compensators. Eventually, the Lyapunov approach ensures that the entire closed-loop BCTTC system is asymptotically stable, and trajectory tracking errors together with other signals are uniformly ultimately bounded.

The rest of this paper is organized as follows. In Section II, the QUAV dynamics and problem formulation are addressed. Backpropagating constraints on intermediate signals are derived in Section III. The BCTTC scheme for trajectory tracking of a QUAV and stability analysis are presented in Sections IV and V, respectively. Simulation studies are conducted in Section VI. Conclusions are drawn in Section VII.

Nomenclature: Throughout this paper, “\| $\cdot \|$ " denotes Euclidean vector norm or Frobenius matrix norm, respectively, and a saturation function sat $(\cdot)$ shown in Fig. 1 is defined by

$$
\operatorname{sat}\left(x ; x_{0}, \delta_{x}\right)= \begin{cases}x, & \left|x-x_{0}\right| \leq \delta_{x} \\ x_{0}+\delta_{x}, & x-x_{0}>\delta_{x} \\ x_{0}-\delta_{x}, & x-x_{0}<-\delta_{x}\end{cases}
$$

where $x_{0}$ and $\delta_{x}>0$ are the center point and range of the saturation, and a smooth approximation to (1) is defined by

$$
\operatorname{sat}_{a}\left(x ; x_{0}, \delta_{x}\right)=x_{0}+\delta_{x} \cdot \tanh \left(\left(x-x_{0}\right) / \delta_{x}\right)
$$

with hyperbolic tangent function $\tanh (\cdot)$. Accordingly, the saturation approximation error function is defined as follows:

$$
\operatorname{sat}_{e}\left(x ; x_{0}, \delta_{x}\right)=\operatorname{sat}\left(x ; x_{0}, \delta_{x}\right)-\operatorname{sat}_{a}\left(x ; x_{0}, \delta_{x}\right)
$$




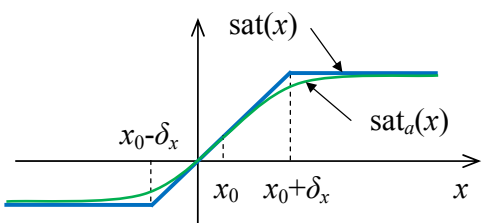

Fig. 1. Saturation function $\operatorname{sat}(\cdot)$ and its smooth approximation $\operatorname{sat}_{a}(\cdot)$.

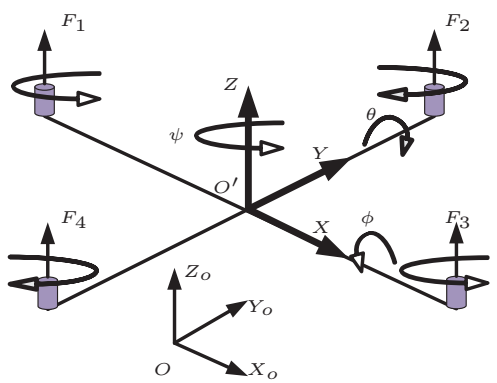

Fig. 2. The configuration of a QUAV.

which is obviously bounded.

\section{QUAV Dynamics AND PRoblem Formulation}

\section{A. QUAV Dynamics}

As shown in Fig. 2, a QUAV is made up of four electric motors fixed on an X-shape frame. The earth-fixed coordinate $O X_{o} Y_{o} Z_{o}$ and the body-fixed coordinate $O^{\prime} X Y Z$ are considered with the origin coinciding to the gravity center of the QUAV. In the earth-fixed frame, the $Z_{0}$-axis points upwards, and the QUAV position is given by a vector $[x, y, z]^{T}$. The QUAV orientation refers to as roll, pitch, and yaw, and is given by the vector $[\phi, \theta, \psi]^{T}$ which is measured with respect to the earth-fixed coordinate. Actually, the entire model of the QUAV is composed by position dynamics, Euler angles, angular velocity, propeller speed and BLDC motor dynamics.

Inspired by the faithful representation for a QUAV with complete dynamics in [58], in this paper, actuator dynamics, i.e., BLDC motor dynamics together with propeller speeds, have been comprehensively incorporated into the entire QUAV dynamics which in turn become much more practical and challenging for controller design and synthesis.

The position dynamics can be described as follows:

$$
\left\{\begin{array}{l}
\dot{\chi}_{11}=\chi_{12} \\
\dot{\chi}_{12}=f_{1}\left(\chi_{12}\right)+u_{1}\left(\chi_{2}, T\left(\chi_{4}\right)\right)+d_{1}
\end{array}\right.
$$

with lumped unknown nonlinearities $\boldsymbol{d}_{1}=\left[d_{11}, d_{12}, d_{13}\right]^{T}$ including model uncertainties, unmodeled dynamics and/or external disturbances which exist in position dynamics, and

$$
\begin{aligned}
\boldsymbol{f}_{1}\left(\boldsymbol{\chi}_{12}\right) & =-\frac{1}{m}\left[\begin{array}{c}
D_{x} \dot{x}^{2} \\
D_{y} \dot{y}^{2} \\
D_{z} \dot{z}^{2}+g
\end{array}\right] \\
\boldsymbol{u}_{1}\left(\chi_{2}, T\left(\chi_{4}\right)\right) & =\frac{T}{m}\left[\begin{array}{c}
\cos \phi \sin \theta \cos \psi+\sin \phi \sin \psi \\
\cos \phi \sin \theta \sin \psi-\sin \phi \cos \psi \\
\cos \phi \cos \theta
\end{array}\right]
\end{aligned}
$$

where $\chi_{11}=[x, y, z]^{T}$ and $\chi_{12}=[\dot{x}, \dot{y}, \dot{z}]^{T}$ are the vectors of the positions and linear velocities in the earth-fixed frame, respectively, $D_{i}(i=x, y, z)$ represents the air resistance coefficient respectively, $m$ is the mass of the QUAV, $g$ is the acceleration of the gravity, $T$ is the total thrust determined by

$$
T\left(\chi_{4}\right)=\sum_{i=1}^{4} b w_{i}^{2}
$$

here, $b$ is the thrust factor and $\chi_{4}=\left[w_{1}, w_{2}, w_{3}, w_{4}\right]^{T}$ is the vector of propeller rotation speeds, and $\chi_{2}=[\phi, \theta, \psi]^{T}$ is the vector of Euler angles governed by

$$
\dot{\chi}_{2}=G_{2}\left(\chi_{2}\right) \boldsymbol{u}_{2}\left(\chi_{3}\right)+\boldsymbol{d}_{2}
$$

with lumped nonlinearities $\boldsymbol{d}_{2}=\left[d_{21}, d_{22}, d_{23}\right]^{T}$ which may include measurement noises and/or external disturbances pertaining to angular velocities, and

$$
\begin{aligned}
& \boldsymbol{G}_{2}\left(\boldsymbol{\chi}_{2}\right)=\left[\begin{array}{ccc}
1 & \sin \phi \tan \theta & \cos \phi \tan \theta \\
0 & \cos \phi & -\sin \phi \\
0 & \frac{\sin \phi}{\cos \theta} & \frac{\cos \phi}{\cos \theta}
\end{array}\right] \\
& \boldsymbol{u}_{2}\left(\boldsymbol{\chi}_{3}\right)=\boldsymbol{\chi}_{3}
\end{aligned}
$$

where $\chi_{3}=[p, q, r]^{T}$ is the angular velocity vector in bodyfixed coordinate given by the following dynamics:

$$
\dot{\chi}_{3}=f_{3}\left(\chi_{3}\right)+G_{3} u_{3}\left(\chi_{4}\right)+d_{3}
$$

with the diagonal matrix $\boldsymbol{G}_{3}=\operatorname{diag}\left(1 / J_{x}, 1 / J_{y}, 1 / J_{z}\right)$ where $J_{i}(i=x, y, z)$ is the moment of inertia with respect to each axis, $\boldsymbol{d}_{3}=\left[d_{31}, d_{32}, d_{33}\right]^{T}$ are unknown nonlinearities within the input channel of angular dynamics, and

$$
\begin{aligned}
\boldsymbol{f}_{3}\left(\boldsymbol{\chi}_{3}\right)= & {\left[\begin{array}{c}
\frac{J_{y}-J_{z}}{J_{x}} q r \\
\frac{J_{z}-J_{x}}{J_{y}} p r \\
\frac{J_{x}-J_{y}}{J_{z}} p q
\end{array}\right] } \\
\boldsymbol{u}_{3}\left(\boldsymbol{\chi}_{4}\right)= & {\left[\begin{array}{c}
l b\left(-w_{2}^{2}+w_{4}^{2}\right) \\
l b\left(-w_{1}^{2}+w_{3}^{2}\right) \\
k\left(-w_{1}^{2}+w_{2}^{2}-w_{3}^{2}+w_{4}^{2}\right)
\end{array}\right] }
\end{aligned}
$$

where the virtual control input $\boldsymbol{u}_{3}\left(\boldsymbol{\chi}_{4}\right)$ is actually constrained by the nonnegative squares, i.e., $w_{1}^{2}, w_{2}^{2}, w_{3}^{2}$ and $w_{4}^{2}, \tau_{i}^{a}(i=$ $1,2,3)$ denotes the airframe torque, $l$ is the distance from the gravity center of QUAV to the propeller rotor, $b$ is the thrust factor, $k$ is the drag factor, and the dynamics of propeller speeds $\chi_{4}=\left[w_{1}, w_{2}, w_{3}, w_{4}\right]^{T}$ are given by

$$
\dot{\chi}_{4}=f_{4}\left(\chi_{4}\right)+G_{4} u_{4}\left(\chi_{5}\right)+d_{4}
$$

with $\boldsymbol{G}_{4}=\boldsymbol{I}_{4} / I_{r}$ where $\boldsymbol{I}_{4} \in R^{4}$ is an unity matirx, $I_{r}$ denotes the propeller rotor inertia, $\boldsymbol{d}_{4}=\left[d_{41}, d_{42}, d_{43}, d_{44}\right]^{T}$ are lumped unknowns for propeller rotation dynamics, and

$$
\begin{aligned}
& \boldsymbol{f}_{4}\left(\boldsymbol{\chi}_{4}\right)=\frac{1}{I_{r}}\left[\begin{array}{c}
-k w_{1}^{2}-c w_{1} \\
-k w_{2}^{2}-c w_{2} \\
-k w_{3}^{2}-c w_{3} \\
-k w_{4}^{2}-c w_{4}
\end{array}\right] \\
& \boldsymbol{u}_{4}\left(\boldsymbol{\chi}_{5}\right):=\left[\tau_{1}, \tau_{2}, \tau_{3}, \tau_{4}\right]^{T}=\frac{n r}{\eta}\left[w_{e 1}^{2}, w_{e 2}^{2}, w_{e 3}^{2}, w_{e 4}^{2}\right]^{T}
\end{aligned}
$$

where the input signal $\boldsymbol{u}_{4}\left(\boldsymbol{\chi}_{5}\right)$ is actually constrained by the nonnegative squares, i.e., $w_{e 1}^{2}, w_{e 2}^{2}, w_{e 3}^{2}$ and $w_{e 4}^{2}, c$ is the thrust factor, $\tau_{i}(i=1,2,3,4)$ denote the thrust torques generated by individual propellers, $n$ is the damping factor, $r$ is the speed 


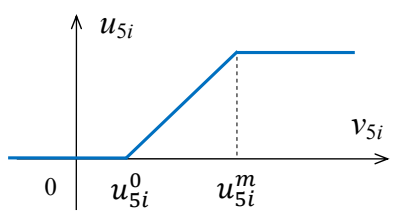

Fig. 3. The input nonlinearity including saturation and dead zone.

ratio between the motor and the propeller, $\eta$ is the transmission efficiency and the dynamics of BLDC motor rotor speeds $\chi_{5}=$ $\left[w_{e 1}, w_{e 2}, w_{e 3}, w_{e 4}\right]^{T}$ is given by

$$
\dot{\chi}_{5}=\boldsymbol{f}_{5}\left(\chi_{5}\right)+\boldsymbol{G}_{5} \boldsymbol{u}_{5}\left(\boldsymbol{v}_{5}\right)+\boldsymbol{d}_{5}
$$

with $\boldsymbol{G}_{5}=C_{m} \boldsymbol{I}_{4} / R_{a}$ where $C_{m}$ and $R_{a}$ denote the electric torque coefficient and the armature resistance of the BLDC motor, respectively, $\boldsymbol{d}_{5}=\left[d_{51}, d_{52}, d_{53}, d_{54}\right]^{T}$ are lumped unknowns pertaining to motor dynamics, $\boldsymbol{u}_{5}\left(\boldsymbol{v}_{5}\right)=$ $\left[u_{51}\left(v_{51}\right), u_{52}\left(v_{52}\right), u_{53}\left(v_{53}\right), u_{54}\left(v_{54}\right)\right]^{T}$ is the armature voltage vector of motors and is practically constrained by input nonlinearities including both saturations and dead zones due to irreversible rotation shown in Fig. 3 as follows:

$$
u_{5 i}\left(v_{5 i}\right)=\operatorname{sat}\left(\mathrm{dz}\left(v_{5 i} ; u_{5 i}^{0}\right) ; \frac{u_{5 i}^{m}-u_{5 i}^{0}}{2}, \frac{u_{5 i}^{m}-u_{5 i}^{0}}{2}\right)
$$

where

$$
\mathrm{dz}\left(v_{5 i} ; u_{5 i}^{0}\right)=v_{5 i}-\operatorname{sat}\left(v_{5 i} ; 0, u_{5 i}^{0}\right)
$$

here, $\operatorname{sat}(\cdot)$ is defined by (1), $u_{5 i}^{0}$ and $u_{5 i}^{m}$ are the dead zone and the saturation of armature voltages, respectively, and $\boldsymbol{v}_{5}=$ $\left[v_{51}, v_{52}, v_{53}, v_{54}\right]^{T}$ is the ideally nominal control input, and

$$
f_{5}\left(\chi_{5}\right)=\frac{1}{J_{r} r^{2}+J_{m} \eta}\left[\begin{array}{l}
-\frac{C_{m} C_{e} \eta}{R_{e}} w_{e 1}-n r w_{e 1}^{2} \\
-\frac{C_{m} C_{e} \eta}{R_{a_{2}}} w_{e 2}-n r w_{e 2}^{2} \\
-\frac{C_{m} C_{e} \eta}{R_{a}} w_{e 3}-n r w_{e 3}^{2} \\
-\frac{C_{m} C_{e} \eta}{R_{a}} w_{e 4}-n r w_{e 4}^{2}
\end{array}\right]
$$

where $J_{r}$ is the moment of inertia of the motor rotor, $J_{m}$ is the inertia moment of the rotating element that turns to rotor of the motor, $C_{e}$ is the voltage coefficient of the motor.

Similar to previous works formulated by Euler angles [32], [36], constraints on Euler angles are naturally required to ensure the nonsingularity of matrix $\boldsymbol{G}_{2}$ in (9) as follows:

Assumption 1. Euler angles are constrained by

$$
\phi, \theta, \psi \in(-\pi / 2, \pi / 2)
$$

Remark 1. For the entire QUAV dynamics (4), (8), (11), (14) and (17), vectorial nonlinearities $\boldsymbol{u}_{1}(\cdot), \boldsymbol{u}_{2}(\cdot), \boldsymbol{u}_{3}(\cdot)$ and $\boldsymbol{u}_{4}(\cdot)$ are taken as virtual control inputs while the signal $u_{5}$ is referred to as the actual control input. As a consequence, a vectorial pure-feedback nonlinear system with interconnected nonlinearities can be innovatively established and is ready for backstepping-like controller design.

Remark 2. In view of the squares of propeller and rotor speeds, i.e., $w_{i}^{2}$ and $w_{e i}^{2}$ in (13) and (16), respectively, together with (7), virtual control signals $\boldsymbol{u}_{1}(\cdot), \boldsymbol{u}_{3}(\cdot)$ and $\boldsymbol{u}_{4}(\cdot)$ in (4), (11) and (14) respectively are expected to be constrained for ensuring the positiveness and boundedness of speed squares. In addition, actuator dynamics with complex constraints arising from insensitive dead-zone voltages, bounded armature voltages and nonreversible rotations have been completely formulated in (17)-(19), and thereby leading to constraints on control input nonlinearity $\boldsymbol{u}_{5}(\cdot)$. To our best knowledge, all aforementioned concerns on backpropagating cascade constraints and complex actuator dynamics have not been addressed in the literature.

Remark 3. In practice, the armature voltage of a BLDC motor within the QUAV is actually limited within a reasonable range, and is usually nonnegative for unidirectional rotation. In addition, both mismatched and matched complex unknowns $\boldsymbol{d}_{i}, i=1, \cdots, 5$ including unmodeled dynamics, uncertainties, measurement noises and external disturbances are incorporated into the QUAV model.

\section{B. Problem Formulation}

In this paper, we address the trajectory tracking problem of a QUAV with backpropagating cascade constraints, complex actuator dynamics and mismatched unknowns within the entire dynamics (4), (8), (11), (14) and (17). Our objective is to design a backpropagating constraints based trajectory tracking controller (BCTTC) such that the complex QUAV can track the desired trajectories under mild conditions as follows:

Assumption 2. The desired trajectory $\left(\chi_{11 d}:=\left[x_{d}, y_{d}, z_{d}\right]^{T}\right.$ and $\left.\psi_{d}\right)$ and its time derivatives are bounded.

Assumption 3. Complex unknowns $\boldsymbol{d}_{i}$ are bounded while the upper bound is unnecessarily known, i.e.,

$$
\left\|\boldsymbol{d}_{i}\right\| \leq L_{i}, \quad i=1,2,3,4,5
$$

where positive constants $L_{i}>0$ is unknown.

In practice, BLDC motors within a QUAV are expected to rotate unidirectionally and generate uniformly upward thrust.

Assumption 4. BLDC motors are nonreversible, i.e., $w_{e i} \geq 0$.

In order to facilitate stability analysis of the closed-loop control system, a preliminary result is stated here.

Lemma 1. Consider the following system:

$$
\dot{x}(t)+\lambda(t) x(t)=\sigma(t)
$$

with $\lambda(t)>0, \forall t$, if $\sigma(t)$ is uniformly bounded, i.e., $|\sigma(t)| \leq$ $\varrho, \forall t$ with a positive constant $\varrho>0$, then states $x(t)$ and $\dot{x}(t)$ are uniformly bounded.

Proof: Consider the Lyapunov function $W=\frac{1}{2} x^{2}$. Using (23) yields the time derivative of $W$ as follows:

$$
\begin{aligned}
\dot{W} & =x(-\lambda x+\sigma) \\
& \leq-(\lambda-\kappa) x^{2}+\frac{\sigma^{2}}{4 \kappa}
\end{aligned}
$$

for any positive constant $\kappa>0$. Since $|\sigma(t)| \leq \varrho, \forall t$, selecting $\kappa<\lambda$, we further have

$$
\dot{W} \leq-a W+b
$$

with $a=2(\lambda-\kappa)$ and $b=\varrho^{2} /(4 \kappa)$. 
It implies that

$$
0 \leq W(t) \leq W(0) e^{-a t}+\left(1-e^{-a t}\right) \frac{b}{a}<\infty
$$

Clearly, $x(t)$ is uniformly bounded (UB), i.e., $|x(t)| \leq \bar{x}, \forall t$. From (23), we further have

$$
|\dot{x}(t)| \leq \lambda \bar{x}+\varrho<\infty, \quad \forall t
$$

which yields $\dot{x}(t)$ is also UB. This concludes the proof.

Remark 4. Unlike previous works, a servo motor control loop is incorporated in this paper, and renders cascade constraints on intermediate signals actually backpropagate from complex actuator dynamics in addition to mismatched unknowns. In this context, an innovative backpropagating cascade constraints based control scheme for such a complex QUAV is established in the sequel.

\section{BACKPROPAGATING CONSTRAINTS}

In order to facilitate our control scheme, backpropagating constraints $(\mathrm{BC})$ on intermediate signals are extracted from nonreversible actuator dynamics and saturations. Key results are summarized as follows:

Proposition 1. The following BC-based saturations hold:

$$
\begin{aligned}
& w_{e i}=\operatorname{sat}\left(w_{e i}^{u} ; w_{e i}^{m} / 2, w_{e i}^{m} / 2\right) \\
& w_{i}=\operatorname{sat}\left(w_{i}^{u} ; w^{m} / 2, w^{m} / 2\right) \\
& T=\operatorname{sat}\left(T^{u} ; 2 b w^{m 2}, 2 b w^{m 2}\right) \\
& u_{4 i}=\operatorname{sat}\left(u_{4 i}^{u} ; n r w_{e i}^{m 2} /(2 \eta), n r w_{e i}^{m 2} /(2 \eta)\right) \\
& u_{3 i}=\operatorname{sat}\left(u_{3 i}^{u} ; 0, u_{3 i}^{m}\right) \\
& \begin{cases}u_{1 i} & =\operatorname{sat}\left(u_{1 i}^{u} ; 0,4 b w^{m 2} / m\right), i=1,2 \\
u_{13} & =\operatorname{sat}\left(u_{13}^{u} ; 2 b w^{m 2} / m, 2 b w^{m 2} / m\right)\end{cases}
\end{aligned}
$$

where sat(.) is defined in (1), $w_{e i}^{m}$ and $w^{m}$ are maximal rotation speeds of motor rotors and propellers, respectively,

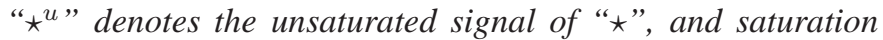
levels for $u_{3 i}$ are given by

$$
\begin{aligned}
& u_{33}^{m}=\frac{k}{b} \min \left\{T, 4 b w^{m^{2}}-T\right\} \\
& u_{32}^{m}=\frac{l}{2} \min \left\{T-\frac{b}{k} u_{33}, 4 b w^{m 2}-T+\frac{b}{k} u_{33}\right\} \\
& u_{31}^{m}=\frac{l}{2} \min \left\{T+\frac{b}{k} u_{33}, 4 b w^{m 2}-T-\frac{b}{k} u_{33}\right\}
\end{aligned}
$$

Proof: Rewriting actuator dynamics (17) as follows:

$$
\dot{w}_{e i}=-a_{i} w_{e i}+b_{i}
$$

with

$$
\begin{aligned}
a_{i} & =\frac{1}{J_{r} r^{2}+J_{m} \eta}\left(\frac{C_{m} C_{e} \eta}{R_{a}}+n r w_{e i}\right) \\
b_{i} & =\frac{C_{m}}{R_{a}} u_{5 i}\left(v_{5 i}\right)+d_{5 i}
\end{aligned}
$$

Together with Assumptions 3-4 and (18), we have

$$
a_{i} \geq \frac{C_{m} C_{e} \eta}{R_{a}\left(J_{r} r^{2}+J_{m} \eta\right)}>0, \quad \forall t
$$

$$
\left|b_{i}\right| \leq \frac{C_{m}}{R_{a}}\left(u_{5 i}^{m}-u_{5 i}^{0}\right)+L_{i}<\infty, \quad \forall t
$$

Using Lemma 1, we immediately have rotor rotation $w_{e i}$ is UB, i.e., $0 \leq w_{e i} \leq w_{e i}^{m}, \forall t$. Similarly, using (14), we have propeller speed $w_{i}$ is $\mathrm{UB}$, i.e., $0 \leq w_{i} \leq w^{m}, \forall t$. Together with (7) and (16), respectively, we have constraints on $T$ and $\boldsymbol{u}_{4}$, i.e., $0 \leq T \leq 4 b w^{m^{2}}$ and $0 \leq u_{4 i} \leq n r w_{e i}^{m 2} / \eta$. In this context, we have (28)-(31) hold.

Together with (7) and (13), we have

$$
\left\{\begin{array}{l}
w_{1}^{2}=-\frac{1}{2 l b} u_{32}-\frac{1}{4 k} u_{33}+\frac{1}{4 b} T \in\left[0, w^{m 2}\right] \\
w_{2}^{2}=-\frac{1}{2 l b} u_{31}+\frac{1}{4 k} u_{33}+\frac{1}{4 b} T \in\left[0, w^{m 2}\right] \\
w_{3}^{2}=\frac{1}{2 l b} u_{32}-\frac{1}{4 k} u_{33}+\frac{1}{4 b} T \in\left[0, w^{m 2}\right] \\
w_{4}^{2}=\frac{1}{2 l b} u_{31}+\frac{1}{4 k} u_{33}+\frac{1}{4 b} T \in\left[0, w^{m 2}\right]
\end{array}\right.
$$

which yields

$$
\begin{aligned}
u_{33} \in \frac{k}{b} & {\left[-\left(4 b w^{m^{2}}-T\right), T\right] \cup \frac{k}{b}\left[-T, 4 b w^{m^{2}}-T\right] } \\
u_{32} \in \frac{l}{2} & {\left[-\left(T-\frac{b}{k} u_{33}\right), 4 b w^{m^{2}}-\left(T-\frac{b}{k} u_{33}\right)\right] } \\
\cup & \frac{l}{2}\left[-\left(4 b w^{m 2}-\left(T-\frac{b}{k} u_{33}\right)\right), T-\frac{b}{k} u_{33}\right] \\
u_{31} \in \frac{l}{2} & {\left[-\left(T+\frac{b}{k} u_{33}\right), 4 b w^{m 2}-\left(T+\frac{b}{k} u_{33}\right)\right] } \\
\cup & \frac{l}{2}\left[-\left(4 b w^{m 2}-\left(T+\frac{b}{k} u_{33}\right)\right), T+\frac{b}{k} u_{33}\right]
\end{aligned}
$$

It follows that saturation constraints on $\boldsymbol{u}_{3}$, i.e., (32) and (34)(36), hold.

Using (6) yields

$$
\begin{aligned}
& u_{11}=\frac{T}{m} \cos (\psi-\delta) \sqrt{\cos ^{2} \phi \sin ^{2} \theta+\sin ^{2} \phi} \\
& u_{12}=\frac{T}{m} \sin (\psi-\delta) \sqrt{\cos ^{2} \phi \sin ^{2} \theta+\sin ^{2} \phi} \\
& u_{13}=\frac{T}{m} \cos \phi \cos \theta
\end{aligned}
$$

with $\delta=\tan ^{-1}(\tan \phi / \sin \theta)$. Together with Assumption 1, we have $\left|u_{11}\right| \leq 4 b w^{m 2} / m,\left|u_{12}\right| \leq 4 b w^{m 2} / m$ and $0 \leq$ $u_{13} \leq 4 b w^{m 2} / m$. In this context, saturations on $\boldsymbol{u}_{1}$ in (33) hold. This concludes the proof.

Proposition 2. Consider desired signals $\boldsymbol{u}_{j d}, j=1,3,4$ of BC-based saturations $\boldsymbol{u}_{j}$ in (31)-(33) defined as follows:

$$
\begin{aligned}
& u_{4 i d}=\operatorname{sat}\left(v_{4 i d} ; n r w_{e i}^{m 2} /(2 \eta), n r w_{e i}^{m 2} /(2 \eta)\right) \\
& u_{3 i d}=\operatorname{sat}\left(v_{3 i d} ; 0, u_{3 i}^{m}\right) \\
& \left\{\begin{array}{l}
u_{1 i d}=\operatorname{sat}\left(v_{1 i d} ; 0,4 b w^{m 2} / m\right), i=1,2 \\
u_{13 d}=\operatorname{sat}\left(v_{13 d} ; 2 b w^{m^{2}} / m, 2 b w^{m 2} / m\right)
\end{array}\right.
\end{aligned}
$$

where $v_{j i d}$ 's are corresponding unsaturated signals. Then, the error $\boldsymbol{u}_{j e}:=\boldsymbol{u}_{j}-\boldsymbol{u}_{j d}$ is bounded, i.e.,

$$
\left\|\boldsymbol{u}_{j e}\right\| \leq \varsigma_{j}
$$

for an unnecessarily known constant $\varsigma_{j}>0$ depending on the saturation level. 


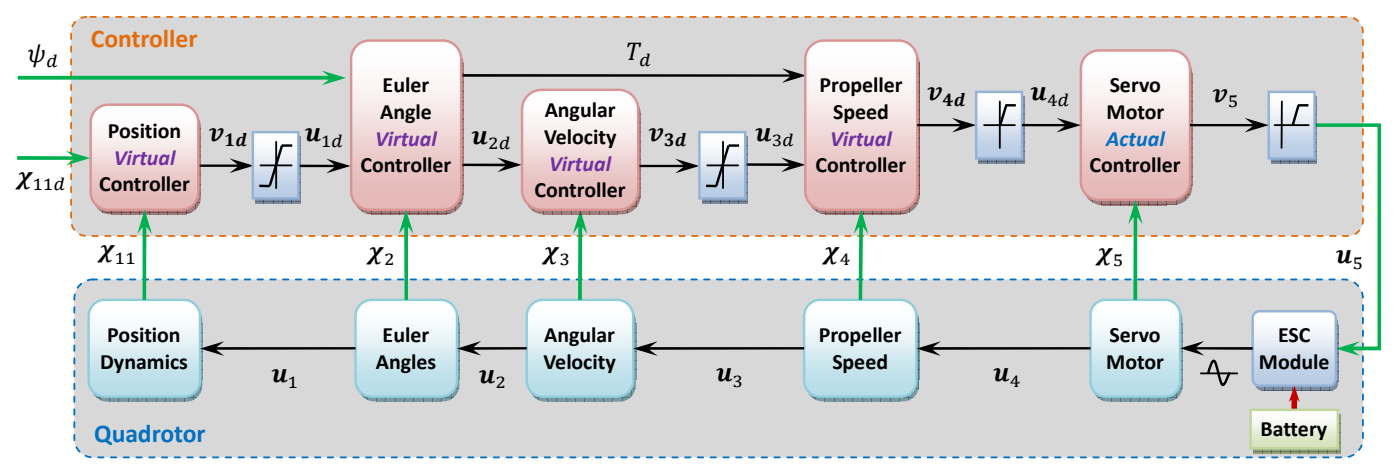

Fig. 4. The overall BCTTC scheme of a quadrotor.

Proof: Combining with (31)-(33) and (49)-(51), we have

$$
\begin{aligned}
\left|u_{4 i e}\right| & \leq n r w_{e i}^{m 2} / \eta \\
\left|u_{3 i e}\right| & \leq 2 u_{3 i}^{m} \\
\left|u_{11 e}\right| & \leq 8 b w^{m 2} / m \\
\left|u_{12 e}\right| & \leq 8 b w^{m^{2}} / m \\
\left|u_{13 e}\right| & \leq 4 b w^{m^{2}} / m
\end{aligned}
$$

This concludes the proof.

Remark 5. Proposition 1 reveals that actuator constraints in (28)-(30) backpropagate recursively to preceding intermediate signals saturated in (31)-(33), and establishes recursive saturation levels which facilitate the BC-based backstepping-like control. Proposition 2 implies that virtual control discrepancies are bounded if desired signals are governed by (49)-(51).

\section{BACKPRopagating CONSTRAints BASED TrajeCtORY TRACKING CONTROL SCHEME}

In this section, the BC-based trajectory tracking control (BCTTC) scheme for a complex QUAV is elaborately established, in a recursive form, by employing an SMC-based DSC framework with universal adaptive compensators for saturation and robustness. As shown in Fig. 4, the entire BCTTC scheme consists of 5 successive controllers, whereby the preceding control effort is used as the desired signal of the succeeding inner closed-loop. Hence, a cascade backstepping-like control hierarchy is synthesized.

\section{A. Position Virtual Controller}

By Proposition 2, the desired position virtual controller (PVC) $\boldsymbol{u}_{1 d}\left(\boldsymbol{v}_{1 d}\right):=\left[u_{11 d}\left(v_{11 d}\right), u_{12 d}\left(v_{12 d}\right), u_{13 d}\left(v_{13 d}\right)\right]^{T}$ is saturated as (51), where $\boldsymbol{v}_{1 d}:=\left[v_{11 d}, v_{12 d}, v_{13 d}\right]^{T}$ is the ideally desired PVC determined later.

Note that the saturated signals $\boldsymbol{u}_{1 d}\left(\boldsymbol{v}_{1 d}\right)$ defined in (51) are non-smooth. In order to facilitate a differentiable PVC, a smooth function $\boldsymbol{g}_{1}\left(\boldsymbol{v}_{1 d}\right)=\left[g_{11}\left(v_{11 d}\right), g_{12}\left(v_{12 d}\right), g_{13}\left(v_{13 d}\right)\right]^{T}$ is devised to approximate the saturated input $\boldsymbol{u}_{1 d}\left(\boldsymbol{v}_{1 d}\right)$ as follows:

$$
\left\{\begin{array}{l}
g_{1 i d}=\operatorname{sat}_{a}\left(v_{1 i d} ; 0,4 b w^{m 2} / m\right), i=1,2 \\
g_{13 d}=\operatorname{sat}_{a}\left(v_{13 d} ; 2 b w^{m 2} / m, 2 b w^{m 2} / m\right)
\end{array}\right.
$$

where $\operatorname{sat}_{a}(\cdot)$ is defined in (2).
Accordingly, the saturation approximation error $\varpi_{1}:=$ $\left[\varpi_{11}, \varpi_{12}, \varpi_{13}\right]^{T}$ is given by

$$
\left\{\begin{array}{l}
\varpi_{1 i}=\operatorname{sat}_{e}\left(v_{1 i d} ; 0,4 b w^{m 2} / m\right), i=1,2 \\
\varpi_{13}=\operatorname{sat}_{e}\left(v_{13 d} ; 2 b w^{m 2} / m, 2 b w^{m 2} / m\right)
\end{array}\right.
$$

where $\operatorname{sat}_{e}(\cdot)$ is defined in (3). Obviously, $\varpi_{1}$ is bounded, i.e.,

$$
\left\|\varpi_{1}\right\| \leq \bar{w}_{1}
$$

here, $\bar{w}_{1}>0$ is unknown.

Define an intermediate tracking error as follows:

$$
\tilde{\boldsymbol{u}}_{1 e}=\boldsymbol{u}_{1}-\boldsymbol{g}_{1}
$$

Using (52) and (60), we immediately have

$$
\left\|\tilde{\boldsymbol{u}}_{1 e}\right\| \leq \rho_{1}
$$

with an unknown upper bound $\rho_{1}=\varsigma_{1}+\bar{w}_{1}$.

Given a reference trajectory $\chi_{11 d}:=\left[x_{d}, y_{d}, z_{d}\right]^{T}$, combining with position dynamics (4), we define the following errors:

$$
\begin{aligned}
\boldsymbol{e}_{11} & =\chi_{11}-\chi_{11 d} \\
\boldsymbol{e}_{12} & =\chi_{12}-\bar{\chi}_{12 d}-\boldsymbol{\mu}_{1} \\
\boldsymbol{y}_{1} & =\bar{\chi}_{12 d}+\boldsymbol{\mu}_{1}-\chi_{12 d}
\end{aligned}
$$

where $\boldsymbol{\mu}_{1}$ is a dynamic compensator determined later, $\chi_{12 d}$ is a virtual control signal, $\bar{\chi}_{12 d}$ is the filtered output of $\chi_{12 d}$ given by

$$
\epsilon_{1} \dot{\bar{\chi}}_{12 d}+\bar{\chi}_{12 d}=\chi_{12 d}
$$

here, $\epsilon_{1}>0$ is an user-defined filtering time constant.

Design sliding surfaces as follows:

$$
\boldsymbol{s}_{1 i}(t)=\boldsymbol{e}_{1 i}(t)+\boldsymbol{K}_{1 i} \int_{0}^{t} \boldsymbol{e}_{1 i}(\tau) d \tau, i=1,2
$$

where $\boldsymbol{K}_{1 i}=\operatorname{diag}\left(k_{1 i 1}, k_{1 i 2}, k_{1 i 3}\right)>0$.

In this context, the virtual control signal $\chi_{12 d}$ can be selected as follows:

$$
\chi_{12 d}=-\boldsymbol{P}_{11} \boldsymbol{s}_{11}+\dot{\chi}_{11 d}-\boldsymbol{K}_{11} \boldsymbol{e}_{11}-\boldsymbol{e}_{12}
$$

where $\boldsymbol{P}_{11}=\operatorname{diag}\left(p_{111}, p_{112}, p_{113}\right)>0$ and an ideally desired PVC for sub-system (4) can be designed as follows:

$$
\begin{aligned}
\boldsymbol{v}_{1 d}= & -\boldsymbol{P}_{12} \boldsymbol{s}_{12}-\boldsymbol{f}_{1}\left(\boldsymbol{\chi}_{12}\right)+\dot{\bar{\chi}}_{12 d} \\
& -\boldsymbol{\mu}_{1}-\boldsymbol{K}_{12} \boldsymbol{e}_{12}-\left(\hat{L}_{1}+\hat{\rho}_{1}\right) \tanh \left(\frac{\boldsymbol{s}_{12}}{\varepsilon}\right)
\end{aligned}
$$


with a positive constant $\varepsilon>0$, and universal adaptive compensators (UAC) for $\mu_{1}, \hat{L}_{1}$ and $\hat{\rho}_{1}$ given by

$$
\left\{\begin{array}{l}
\dot{\boldsymbol{\mu}}_{1}=-\boldsymbol{\mu}_{1}+\boldsymbol{g}_{1}\left(\boldsymbol{v}_{1 d}\right)-\boldsymbol{v}_{1 d} \\
\dot{\hat{L}}_{1}=-\gamma_{11} \hat{L}_{1}+\kappa_{L, 1} \boldsymbol{s}_{12}^{T} \tanh \left(\frac{\boldsymbol{s}_{12}}{\varepsilon}\right) \\
\dot{\hat{\rho}}_{1}=-\gamma_{12} \hat{\rho}_{1}+\kappa_{\rho, 1} \boldsymbol{s}_{12}^{T} \tanh \left(\frac{\boldsymbol{s}_{12}}{\varepsilon}\right)
\end{array}\right.
$$

where $\boldsymbol{P}_{12}=\operatorname{diag}\left(p_{121}, p_{122}, p_{123}\right)>0, \gamma_{11}>0, \gamma_{12}>0$, $\kappa_{L, 1}>0, \kappa_{\rho, 1}>0$, and $\hat{L}_{1}$ and $\hat{\rho}_{1}$ are estimates of unknown bounds $L_{1}$ and $\rho_{1}$, respectively.

In this context, the sliding error dynamics can be obtained as follows:

$$
\begin{aligned}
& \dot{\boldsymbol{s}}_{11}=-\boldsymbol{P}_{11} \boldsymbol{s}_{11}+\boldsymbol{y}_{1} \\
& \dot{\boldsymbol{s}}_{12}=-\boldsymbol{P}_{12} \boldsymbol{s}_{12}+\boldsymbol{d}_{1}+\tilde{\boldsymbol{u}}_{1 e}-\left(\hat{L}_{1}+\hat{\rho}_{1}\right) \tanh \left(\frac{\boldsymbol{s}_{12}}{\varepsilon}\right)
\end{aligned}
$$

with $\tilde{\boldsymbol{u}}_{1 e}$ defined in (61).

\section{B. Euler Angle Virtual Controller}

Substituting (51) into the input nonlinearity (6) yields

$$
\left\{\begin{array}{l}
\mathcal{T}_{d}\left(\cos \phi_{d} \sin \theta_{d} \cos \psi_{d}+\sin \phi_{d} \sin \psi_{d}\right)=m u_{11 d} \\
\mathcal{T}_{d}\left(\cos \phi_{d} \sin \theta_{d} \sin \psi_{d}-\sin \phi_{d} \cos \psi_{d}\right)=m u_{12 d} \\
\mathcal{T}_{d} \cos \phi_{d} \cos \theta_{d}=m u_{13 d}
\end{array}\right.
$$

Given a reference yaw angle $\psi_{d}$, applying a direct calculation to (73) and using (30) in Proposition 1, we have

$$
\left\{\begin{array}{l}
T_{d}=\operatorname{sat}\left(\mathcal{T}_{d} ; 2 b w^{m 2}, 2 b w^{m 2}\right) \\
\phi_{d}=\arcsin \left(\frac{m}{\mathcal{T}_{d}}\left(\sin \psi_{d} u_{11 d}-\cos \psi_{d} u_{12 d}\right)\right) \\
\theta_{d}=\arcsin \left(\frac{\frac{\frac{T}{d}_{d}}{\mathcal{L}_{11 d}}-\sin \psi_{d} \sin \phi_{d}}{\cos \psi_{d} \cos \phi_{d}}\right)
\end{array}\right.
$$

where $\mathcal{T}_{d}=m\left\|\boldsymbol{u}_{1 d}\right\|$ and $\boldsymbol{u}_{1 d}:=\left[u_{11 d}, u_{12 d}, u_{13 d}\right]^{T}$.

Let $\chi_{2 d}:=\left[\phi_{d}, \theta_{d}, \psi_{d}\right]^{T}$ and $\bar{\chi}_{2 d}:=\left[\bar{\phi}_{d}, \bar{\theta}_{d}, \bar{\psi}_{d}\right]^{T}$ where $\bar{\chi}_{2 d}$ is the filtered output of $\chi_{2 d}$ given by

$$
\epsilon_{2} \dot{\bar{\chi}}_{2 d}+\bar{\chi}_{2 d}=\chi_{2 d}
$$

here, $\epsilon_{2}>0$ is an user-defined filtering time constant.

Combining with Euler angles dynamics (8), we define the following errors:

$$
\begin{aligned}
& \boldsymbol{e}_{2}=\chi_{2}-\bar{\chi}_{2 d} \\
& \boldsymbol{y}_{2}=\bar{\chi}_{2 d}-\chi_{2 d}
\end{aligned}
$$

Design a sliding surface as follows:

$$
\boldsymbol{s}_{2}(t)=\boldsymbol{e}_{2}(t)+\boldsymbol{K}_{2} \int_{0}^{t} \boldsymbol{e}_{2}(\tau) d \tau
$$

where $\boldsymbol{K}_{2}=\operatorname{diag}\left(k_{21}, k_{22}, k_{23}\right)>0$.

In this context, the desired Euler angle virtual controller (EAVC) for sub-system (8) can be designed as follows:

$$
\begin{aligned}
\boldsymbol{u}_{2 d}=\boldsymbol{G}_{2}^{-1}\left(\boldsymbol{\chi}_{2}\right)[ & \dot{\bar{\chi}}_{2 d}-\boldsymbol{K}_{2} \boldsymbol{e}_{2} \\
& \left.-\boldsymbol{P}_{2} \boldsymbol{s}_{2}+\beta_{2} \boldsymbol{y}_{2}-\hat{L}_{2} \tanh \left(\frac{\boldsymbol{s}_{2}}{\varepsilon}\right)\right]
\end{aligned}
$$

with an UAC $\hat{L}_{2}$ given by

$$
\dot{\hat{L}}_{2}=-\gamma_{21} \hat{L}_{2}+\kappa_{L, 2} \boldsymbol{s}_{2}^{T} \tanh \left(\frac{\boldsymbol{s}_{2}}{\varepsilon}\right)
$$

where $\boldsymbol{P}_{2}=\operatorname{diag}\left(p_{21}, p_{22}, p_{23}\right)>0, \beta_{2}>0, \gamma_{21}>0, \kappa_{L, 2}>$ 0 , and $\hat{L}_{2}$ is the estimate of unknown bound $L_{2}$.

Hence, the sliding error dynamics can be obtained as follows:

$$
\dot{\boldsymbol{s}}_{2}=-\boldsymbol{P}_{2} \boldsymbol{s}_{2}+\boldsymbol{d}_{2}+\boldsymbol{G}_{2} \boldsymbol{u}_{2 e}+\beta_{2} \boldsymbol{y}_{2}-\hat{L}_{2} \tanh \left(\frac{\boldsymbol{s}_{2}}{\varepsilon}\right)
$$

where

$$
\boldsymbol{u}_{2 e}=\boldsymbol{u}-\boldsymbol{u}_{2 d}=\chi_{3}-\chi_{3 d}
$$

Remark 6. The derivation of (74) from (73) can be obtained by assigning a given reference yaw angle $\psi_{d}$. In addition, the first equation of (74) ensures the desired total thrust $T_{d}$ is reasonable, whereby possible saturation can be tackled later.

\section{Angular Velocity Virtual Controller}

The saturated angular velocity virtual controller (AVVC) $\boldsymbol{u}_{3 d}\left(\boldsymbol{v}_{3 d}\right):=\left[u_{31 d}\left(v_{31 d}\right), u_{32 d}\left(v_{32 d}\right), u_{33 d}\left(v_{33 d}\right)\right]^{T}$ is designed as (50) with saturation levels in (34)-(36), where $\boldsymbol{v}_{3 d}:=$ $\left[v_{31 d}, v_{32 d}, v_{33 d}\right]^{T}$ is the ideally desired AVVC determined later.

Note the constrained control input $\boldsymbol{u}_{3 d}\left(\boldsymbol{v}_{3 d}\right)$ defined in (50) and (34)-(36) is non-smooth. In order to facilitate a differentiable virtual control law, a smooth function $\boldsymbol{g}_{3}\left(\boldsymbol{v}_{3 d}\right)=$ $\left[g_{31}\left(v_{31 d}\right), g_{32}\left(v_{32 d}\right), g_{33}\left(v_{33 d}\right)\right]^{T}$ is devised to approximate the constrained input $\boldsymbol{u}_{3 d}\left(\boldsymbol{v}_{3 d}\right)$ as follows:

$$
g_{3 i}\left(v_{3 i d}\right)=\operatorname{sat}_{a}\left(v_{3 i d} ; 0, u_{3 i}^{m}\right)
$$

where $\operatorname{sat}_{a}(\cdot)$ is defined in (2) and $u_{3 i}^{m}$ is given by (34)-(36).

Accordingly, the constraint approximation error $\varpi_{3}:=$ $\left[\varpi_{31}, \varpi_{32}, \varpi_{33}\right]^{T}$ is given by

$$
\varpi_{3 i}=\operatorname{sat}_{e}\left(v_{3 i d} ; 0, u_{3 i}^{m}\right)
$$

where $\operatorname{sat}_{e}(\cdot)$ is defined in (3). Obviously, $\varpi_{3}$ is bounded, i.e.,

$$
\left\|G_{3} \varpi_{3}\right\| \leq \bar{w}_{3}
$$

here, $\bar{w}_{3}>0$ is unknown.

Define an intermediate tracking error as follows:

$$
\tilde{\boldsymbol{u}}_{3 e}=\boldsymbol{u}_{3}-\boldsymbol{g}_{3}
$$

Using (52) and (85), we immediately have

$$
\left\|\boldsymbol{G}_{3} \tilde{\boldsymbol{u}}_{3 e}\right\| \leq \rho_{3}
$$

with an unknown upper bound $\rho_{3}=\left\|\boldsymbol{G}_{3}\right\| \varsigma_{3}+\bar{w}_{3}$.

Together with angular velocity dynamics (11), we design a sliding surface as follows:

$$
\boldsymbol{s}_{3}(t)=\boldsymbol{u}_{2}-\boldsymbol{u}_{2 d}-\boldsymbol{y}_{3}=\boldsymbol{e}_{3}(t)
$$

with

$$
\begin{aligned}
& \boldsymbol{e}_{3}=\chi_{3}-\bar{\chi}_{3 d}-\boldsymbol{\mu}_{3} \\
& \boldsymbol{y}_{3}=\bar{\chi}_{3 d}+\boldsymbol{\mu}_{3}-\chi_{3 d}
\end{aligned}
$$

where $\boldsymbol{\mu}_{3}$ is determined later, $\bar{\chi}_{3 d}:=\left[\bar{p}_{d}, \bar{q}_{d}, \bar{r}_{d}\right]^{T}$ is the filtered output of $\chi_{3 d}:=\left[p_{d}, q_{d}, r_{d}\right]^{T}=\boldsymbol{u}_{2 d}$ and is given by

$$
\epsilon_{3} \dot{\bar{\chi}}_{3 d}+\bar{\chi}_{3 d}=\chi_{3 d}
$$

here, $\epsilon_{3}>0$ is an user-defined filtering time constant. 
Accordingly, an ideally desired AVVC for sub-system (11) can be governed as follows:

$$
\begin{gathered}
\boldsymbol{v}_{3 d}=\boldsymbol{G}_{3}^{-1}\left[\dot{\bar{\chi}}_{3 d}-\boldsymbol{f}_{3}\left(\chi_{3}\right)-\boldsymbol{G}_{2}^{T} \boldsymbol{s}_{2}-\boldsymbol{P}_{3} \boldsymbol{s}_{3}-\boldsymbol{G}_{3} \boldsymbol{\mu}_{3}\right. \\
\left.+\beta_{3} \boldsymbol{y}_{3}-\left(\hat{L}_{3}+\hat{\rho}_{3}\right) \tanh \left(\frac{\boldsymbol{s}_{3}}{\varepsilon}\right)\right]
\end{gathered}
$$

with the UAC for $\boldsymbol{\mu}_{3}, \hat{L}_{3}$ and $\hat{\rho}_{3}$ given by

$$
\left\{\begin{array}{l}
\dot{\mu}_{3}=\boldsymbol{G}_{3}\left(\boldsymbol{g}_{3}\left(\boldsymbol{v}_{3 d}\right)-\boldsymbol{v}_{3 d}-\boldsymbol{\mu}_{3}\right) \\
\dot{\hat{L}}_{3}=-\gamma_{31} \hat{L}_{3}+\kappa_{L, 3} \boldsymbol{s}_{3}^{T} \tanh \left(\frac{\boldsymbol{s}_{3}}{\varepsilon}\right) \\
\dot{\hat{\rho}}_{3}=-\gamma_{32} \hat{\rho}_{3}+\kappa_{\rho, 3} \boldsymbol{s}_{3}^{T} \tanh \left(\frac{\boldsymbol{s}_{3}}{\varepsilon}\right)
\end{array}\right.
$$

where $\boldsymbol{P}_{3}=\operatorname{diag}\left(p_{31}, p_{32}, p_{33}\right)>0, \beta_{3}>0, \gamma_{31}>0, \gamma_{32}>$ $0, \kappa_{L, 3}>0, \kappa_{\rho, 3}>0$, and $\hat{L}_{3}$ and $\hat{\rho}_{3}$ are estimates of unknown bounds $L_{3}$ and $\rho_{3}$, respectively.

In this context, the sliding error dynamics can be obtained as follows:

$$
\begin{aligned}
\dot{\boldsymbol{s}}_{3}=-\boldsymbol{P}_{3} \boldsymbol{s}_{3} & -\boldsymbol{G}_{2}^{T} \boldsymbol{s}_{2}+\boldsymbol{d}_{3} \\
& +\boldsymbol{G}_{3} \tilde{\boldsymbol{u}}_{3 e}+\beta_{3} \boldsymbol{y}_{3}-\left(\hat{L}_{3}+\hat{\rho}_{3}\right) \tanh \left(\frac{\boldsymbol{s}_{3}}{\varepsilon}\right)
\end{aligned}
$$

where $\tilde{\boldsymbol{u}}_{3 e}$ is given by (86).

Remark 7. The saturation of $T_{d}$ in (74) can be transferred to constraints on $\boldsymbol{u}_{3 d}$ given by (50).

\section{Propeller Speed Virtual Controller}

The desired propeller speed virtual controller (PSVC) $\boldsymbol{u}_{4 d}\left(\boldsymbol{v}_{4 d}\right):=\left[u_{41 d}\left(v_{41 d}\right), u_{42 d}\left(v_{42 d}\right), u_{43 d}\left(v_{43 d}\right), u_{44 d}\left(v_{44 d}\right)\right]^{T}$ is designed as (49), where $\boldsymbol{v}_{4 d}:=\left[v_{41 d}, v_{42 d}, v_{43 d}, v_{44 d}\right]^{T}$ is the ideally desired PSVC determined later.

Note the constrained control input $\boldsymbol{u}_{4 d}\left(\boldsymbol{v}_{4 d}\right)$ defined in (49) is non-smooth. In order to facilitate a differentiable virtual control law, a differentiable function $\boldsymbol{g}_{4}\left(\boldsymbol{v}_{4 d}\right):=$ $\left[g_{41}\left(v_{41 d}\right), g_{42}\left(v_{42 d}\right), g_{43}\left(v_{43 d}\right), g_{44}\left(v_{44 d}\right)\right]^{T}$ is employed to approximate the non-smooth constrained input $\boldsymbol{u}_{4 d}\left(\boldsymbol{v}_{4 d}\right)$ as follows:

$$
g_{4 i}\left(v_{4 i d}\right)=\operatorname{sat}_{a}\left(v_{4 i d} ; n r w_{e i}^{m^{2}} /(2 \eta), n r w_{e i}^{m^{2}} /(2 \eta)\right)
$$

where $\operatorname{sat}_{a}(\cdot)$ is defined in (2). The constraint approximation error $\varpi_{4}=\left[\varpi_{41}, \varpi_{42}, \varpi_{43}, \varpi_{44}\right]^{T}$ is determined by

$$
\varpi_{4 i}=\operatorname{sat}_{e}\left(v_{4 i d} ; n r w_{e i}^{m 2} /(2 \eta), n r w_{e i}^{m 2} /(2 \eta)\right)
$$

where $\operatorname{sat}_{e}(\cdot)$ is defined in (3). Obviously, $\varpi_{4}$ is bounded, i.e.,

$$
\left\|G_{4} \varpi_{4}\right\| \leq \bar{w}_{4}
$$

here, $\bar{w}_{4}>0$ is unknown.

Define an intermediate tracking error as follows:

$$
\tilde{\boldsymbol{u}}_{4 e}=\boldsymbol{u}_{4}-\boldsymbol{g}_{4}
$$

Using (52) and (97), we immediately have

$$
\left\|\boldsymbol{G}_{4} \tilde{\boldsymbol{u}}_{4 e}\right\| \leq \rho_{4}
$$

with an unknown upper bound $\rho_{4}=\left\|\boldsymbol{G}_{4}\right\| \varsigma_{4}+\bar{w}_{4}$.

Note that the actually desired control law $\boldsymbol{u}_{3 d}$ can be derived from (49). Together with the following equations deriving from
(7) and (13):

$$
\left\{\begin{array}{l}
w_{1 d}=\left(-\frac{1}{2 l b} u_{32 d}-\frac{1}{4 k} u_{33 d}+\frac{1}{4 b} T_{d}\right)^{1 / 2} \\
w_{2 d}=\left(-\frac{1}{2 l b} u_{31 d}+\frac{1}{4 k} u_{33 d}+\frac{1}{4 b} T_{d}\right)^{1 / 2} \\
w_{3 d}=\left(\frac{1}{2 l b} u_{32 d}-\frac{1}{4 k} u_{33 d}+\frac{1}{4 b} T_{d}\right)^{1 / 2} \\
w_{4 d}=\left(\frac{1}{2 l b} u_{31 d}+\frac{1}{4 k} u_{33 d}+\frac{1}{4 b} T_{d}\right)^{1 / 2}
\end{array}\right.
$$

we can obtain the reference $\chi_{4 d}:=\left[w_{1 d}, w_{2 d}, w_{3 d}, w_{4 d}\right]^{T}$, and the filtered signals $\bar{\chi}_{4 d}:=\left[\bar{w}_{1 d}, \bar{w}_{2 d}, \bar{w}_{3 d}, \bar{w}_{4 d}\right]^{T}$ given by

$$
\epsilon_{4} \dot{\bar{\chi}}_{4 d}+\bar{\chi}_{4 d}=\chi_{4 d}
$$

where $\epsilon_{4}>0$ is an user-defined filtering time constant.

Combining with propeller speed dynamics (14), we define

$$
\begin{aligned}
& \boldsymbol{e}_{4}=\chi_{4}-\bar{\chi}_{4 d}-\boldsymbol{\mu}_{4} \\
& \boldsymbol{y}_{4}=\bar{\chi}_{4 d}+\boldsymbol{\mu}_{4}-\chi_{4 d}
\end{aligned}
$$

where $\boldsymbol{\mu}_{4}$ is determined later.

Design a sliding surface as follows:

$$
\boldsymbol{s}_{4}(t)=\boldsymbol{e}_{4}(t)+\boldsymbol{K}_{4} \int_{0}^{t} \boldsymbol{e}_{4}(\tau) d \tau
$$

with $K_{4}=\operatorname{diag}\left(k_{41}, k_{42}, k_{43}, k_{44}\right)>0$.

In this context, the ideally desired propeller speed control law for sub-system (14) can be designed as follows:

$$
\begin{aligned}
\boldsymbol{v}_{4 d}=\boldsymbol{G}_{4}^{-1}[ & \dot{\bar{\chi}}_{4 d}-\boldsymbol{f}_{4}\left(\boldsymbol{\chi}_{4}\right)-\boldsymbol{K}_{4} \boldsymbol{e}_{4}-\boldsymbol{P}_{4} \boldsymbol{s}_{4} \\
& \left.-\boldsymbol{G}_{4} \boldsymbol{\mu}_{4}+\beta_{4} \boldsymbol{y}_{4}-\left(\hat{L}_{4}+\hat{\rho}_{4}\right) \tanh \left(\frac{\boldsymbol{s}_{4}}{\varepsilon}\right)\right]
\end{aligned}
$$

with the UAC for $\boldsymbol{\mu}_{4}, \hat{L}_{4}$ and $\hat{\rho}_{4}$ given by

$$
\left\{\begin{array}{l}
\dot{\boldsymbol{\mu}}_{4}=\boldsymbol{G}_{4}\left(\boldsymbol{g}_{4}\left(\boldsymbol{v}_{4 d}\right)-\boldsymbol{v}_{4 d}-\boldsymbol{\mu}_{4}\right) \\
\dot{\hat{L}}_{4}=-\gamma_{41} \hat{L}_{4}+\kappa_{L, 4} \boldsymbol{s}_{4}^{T} \tanh \left(\frac{\boldsymbol{s}_{4}}{\varepsilon}\right) \\
\dot{\hat{\rho}}_{4}=-\gamma_{42} \hat{\rho}_{4}+\kappa_{\rho, 4} \boldsymbol{s}_{4}^{T} \tanh \left(\frac{\boldsymbol{s}_{4}}{\varepsilon}\right)
\end{array}\right.
$$

where $\boldsymbol{P}_{4}=\operatorname{diag}\left(p_{41}, p_{42}, p_{43}, p_{44}\right)>0, \beta_{4}>0, \gamma_{41}>0$, $\gamma_{41}>0, \gamma_{42}>0, \kappa_{L, 4}>0, \kappa_{\rho, 4}>0$, and $\hat{L}_{4}$ and $\hat{\rho}_{4}$ are estimates of unknown bounds $L_{4}$ and $\rho_{4}$, respectively.

In this context, the sliding error dynamics can be obtained as follows:

$$
\begin{aligned}
\dot{\boldsymbol{s}}_{4}=-\boldsymbol{P}_{4} \boldsymbol{s}_{4} & +\boldsymbol{d}_{4}+\boldsymbol{G}_{4} \tilde{\boldsymbol{u}}_{4 e} \\
& +\beta_{4} \boldsymbol{y}_{4}-\left(\hat{L}_{4}+\hat{\rho}_{4}\right) \tanh \left(\frac{\boldsymbol{s}_{4}}{\varepsilon}\right)
\end{aligned}
$$

where $\tilde{\boldsymbol{u}}_{4 e}$ is given by (98).

\section{E. Servo Motor Actual Controller}

The actually desired signal $\boldsymbol{u}_{4 d}$ can be derived from (49) and (105). Using (16), we can obtain the desired vector $\chi_{5 d}:=\left[w_{e 1 d}, w_{e 2 d}, w_{e 3 d}, w_{e 4 d}\right]^{T}$, and $\bar{\chi}_{5 d}:=$ $\left[\bar{w}_{e 1 d}, \bar{w}_{e 2 d}, \bar{w}_{e 3 d}, \bar{w}_{e 4 d}\right]^{T}$ is the filtered output given by

$$
\epsilon_{5} \dot{\bar{\chi}}_{5 d}+\bar{\chi}_{5 d}=\chi_{5 d}
$$

here, $\epsilon_{5}>0$ is an user-defined filtering time constant.

Combining with servo motor dynamics (17) and the input nonlinearities (18) and (19), we define the following errors:

$$
e_{5}=\chi_{5}-\bar{\chi}_{5 d}-\mu_{5}
$$




$$
\boldsymbol{y}_{5}=\bar{\chi}_{5 d}+\boldsymbol{\mu}_{5}-\chi_{5 d}
$$

where $\boldsymbol{\mu}_{5}$ is determined later, $\boldsymbol{u}_{5}(\cdot)$ is the nonlinear input constrained by saturation and dead zone in (18) and (19).

Design a sliding surface as follows:

$$
\boldsymbol{s}_{5}(t)=\boldsymbol{e}_{5}(t)+\boldsymbol{K}_{5} \int_{0}^{t} \boldsymbol{e}_{5}(\tau) d \tau
$$

with $\boldsymbol{K}_{5}=\operatorname{diag}\left(k_{51}, k_{52}, k_{53}, k_{54}\right)>0$.

Eventually, the nominal control law, i.e., the servo motor actual controller (SMAC) $\boldsymbol{v}_{5}$, can be designed as follows:

$$
\begin{array}{r}
\boldsymbol{v}_{5}=\boldsymbol{G}_{5}^{-1}\left[\dot{\bar{\chi}}_{5 d}-\boldsymbol{f}_{5}\left(\boldsymbol{\chi}_{5}\right)-\boldsymbol{K}_{5} \boldsymbol{e}_{5}-\boldsymbol{P}_{5} \boldsymbol{s}_{5}\right. \\
\left.-\boldsymbol{G}_{5} \boldsymbol{\mu}_{5}+\beta_{5} \boldsymbol{y}_{5}-\hat{L}_{5} \tanh \left(\frac{\boldsymbol{s}_{5}}{\varepsilon}\right)\right]
\end{array}
$$

with the UAC for $\boldsymbol{\mu}$ and $\hat{L}_{5}$ given by

$$
\left\{\begin{array}{l}
\dot{\boldsymbol{\mu}}_{5}=\boldsymbol{G}_{5}\left(\boldsymbol{u}_{5}\left(\boldsymbol{v}_{5}\right)-\boldsymbol{v}_{5}-\boldsymbol{\mu}_{5}\right) \\
\dot{\hat{L}}_{5}=-\gamma_{51} \hat{L}_{5}+\kappa_{L, 5} \boldsymbol{s}_{5}^{T} \tanh \left(\frac{\boldsymbol{s}_{5}}{\varepsilon}\right)
\end{array}\right.
$$

where $\boldsymbol{P}_{5}=\operatorname{diag}\left(p_{51}, p_{52}, p_{53}, p_{54}\right)>0, \beta_{5}>0, \gamma_{51}>0$, $\kappa_{L, 5}>0$, and $\hat{L}_{5}$ is the estimate of unknown bound $L_{5}$.

Hence, the sliding error dynamics is obtained as follows:

$$
\dot{\boldsymbol{s}}_{5}=-\boldsymbol{P}_{5} \boldsymbol{s}_{5}+\boldsymbol{d}_{5}+\beta_{5} \boldsymbol{y}_{5}-\hat{L}_{5} \tanh \left(\frac{\boldsymbol{s}_{5}}{\varepsilon}\right)
$$

Remark 8. Bounded intermediate errors in (61), (86) and (98) decouple sliding error dynamics (71), (72), (94) and (107), and leave only $\dot{\boldsymbol{s}}_{2}$ in (81) be driven by the input discrepancy $\boldsymbol{u}_{2 e}$ which is closely related with the cascade sliding surface $s_{3}$. In addition, as shown in Fig. 4, the BCTTC scheme is composed by 4 successive virtual sub-controllers in (69), (79), (92) and (105), and 1 actual sub-controller in (112). In this context, each sub-controller for an individual subsystem can be designed independently by using various approaches although the SMC technique is exclusively employed in this paper. In essence, this significant advantage actually benefits from the $B C$-based cutting by virtue of bounded intermediate errors.

Remark 9. Note that the ESC module is still required to be used for generating PWM waves which drive and regulate BLDCs even though actuator dynamics have been completely addressed in the proposed BCTTC scheme. Unlike traditional ESC modules which are open-loop control systems, the closedloop ESC can be achieved in the BCTTC scheme, and thereby enhancing its regulation accuracy and robustness.

Remark 10. Note that the BCTTC scheme only requires a nominal model, and even is a model-free approach if nominal dynamics $f_{i}, i=1, \cdots, 5$ are completely unknown and thereby encapsulating into unknowns $\boldsymbol{d}_{i}$. In addition, nonlinear state observers can also be designed to extend the BCTTC to an output-feedback control approach.

Remark 11. Filters applied to virtual signals might cause high-gain problem pertaining to filter-backstepping (i.e., DSC) or high-gain observer design [59]. In the BCTTC scheme, unexpected magnitudes and/or peaks are actually saturated by $B C$-based constraints. The SMC technique employed in subcontrollers is expected to enhance steady-state tracking accu- racy via incorporating an integral term. Actually, if integral gains $\boldsymbol{K}_{i}$ are chosen as zeros, sliding-mode surfaces degrade to intermediate tracking errors.

Remark 12. From (51), (69), (79), (50), (92), (49), (105) and (112), we can see that the computational complexity of the BCTTC scheme is similar to adaptive approximation based state-feedback approach.

\section{Stability AnAlysis}

A key result on stability analysis is summarized as follows:

Theorem 1. Consider a complex QUAV system (4), (8), (11), (14) and (17), together with the proposed BCTTC scheme (51), (69), (79), (50), (92), (49), (105) and (112) with the UAC given by (70), (80), (93), (106) and (113), tracking errors are uniformly ultimately bounded and all other signals of the closed-loop control system are bounded.

Proof: Consider the following Lyapunov function:

$$
\begin{aligned}
V=\frac{1}{2}\left[\boldsymbol{s}_{11}^{T} \boldsymbol{s}_{11}+\boldsymbol{s}_{12}^{T} \boldsymbol{s}_{12}+\sum_{i=2}^{5} \boldsymbol{s}_{i}^{T} \boldsymbol{s}_{i}+\sum_{i=1}^{5} \boldsymbol{y}_{i}^{T} \boldsymbol{y}_{i}\right. \\
\left.+\sum_{i=1}^{5} \kappa_{L, i}^{-1} \tilde{L}_{i}^{2}+\kappa_{\rho, 1}^{-1} \tilde{\rho}_{1}^{2}+\kappa_{\rho, 3}^{-1} \tilde{\rho}_{3}^{2}+\kappa_{\rho, 4}^{-1} \tilde{\rho}_{4}^{2}\right]
\end{aligned}
$$

with $\tilde{L}_{i}=L_{i}-\hat{L}_{i}, i=1, \cdots, 5, \tilde{\rho}_{1}=\rho_{1}-\hat{\rho}_{1}, \tilde{\rho}_{3}=\rho_{3}-\hat{\rho}_{3}$ and $\tilde{\rho}_{4}=\rho_{4}-\hat{\rho}_{4}$.

Together with (71), (72), (81), (94), (107) and (114), and using (62), (87) and (99), we have the time derivative of $V$ can be derived as follows:

$$
\begin{aligned}
\dot{V} \leq & \sum_{i=2}^{5}\left[-\boldsymbol{s}_{i}^{T} \boldsymbol{P}_{i} \boldsymbol{s}_{i}+\beta_{i} \boldsymbol{s}_{i}^{T} \boldsymbol{y}_{i}+L_{i}\left\|\boldsymbol{s}_{i}\right\|-\hat{L}_{i} \boldsymbol{s}_{i}^{T} \tanh \left(\frac{\boldsymbol{s}_{i}}{\varepsilon}\right)\right] \\
& +\sum_{i=1}^{5}\left(-\kappa_{L, i}^{-1} \tilde{L}_{i} \dot{\hat{L}}_{i}+\boldsymbol{y}_{i}^{T} \dot{\boldsymbol{y}}_{i}\right)+\rho_{1}\left\|\boldsymbol{s}_{12}\right\|+\rho_{3}\left\|\boldsymbol{s}_{3}\right\|+\rho_{4}\left\|\boldsymbol{s}_{4}\right\| \\
& -\kappa_{\rho, 1}^{-1} \tilde{\rho}_{1} \dot{\hat{\rho}}_{1}-\kappa_{\rho, 3}^{-1} \tilde{\rho}_{3} \dot{\hat{\rho}}_{3}-\kappa_{\rho, 4}^{-1} \tilde{\rho}_{4} \dot{\hat{\rho}}_{4}-\hat{\rho}_{1} \boldsymbol{s}_{12}^{T} \tanh \left(\frac{\boldsymbol{s}_{12}}{\varepsilon}\right) \\
& -\hat{\rho}_{3} \boldsymbol{s}_{3}^{T} \tanh \left(\frac{\boldsymbol{s}_{3}}{\varepsilon}\right)-\hat{\rho}_{4} \boldsymbol{s}_{4}^{T} \tanh \left(\frac{\boldsymbol{s}_{4}}{\varepsilon}\right)-\boldsymbol{s}_{11}^{T} \boldsymbol{P}_{11} \boldsymbol{s}_{11} \\
& +\boldsymbol{s}_{11}^{T} \boldsymbol{y}_{1}+\boldsymbol{s}_{2}^{T} \boldsymbol{G}_{2} \boldsymbol{y}_{3}-\boldsymbol{s}_{12}^{T} \boldsymbol{P}_{12} \boldsymbol{s}_{12}+L_{1}\left\|\boldsymbol{s}_{12}\right\| \\
& -\hat{L}_{1} \boldsymbol{s}_{12}^{T} \tanh \left(\frac{\boldsymbol{s}_{12}}{\varepsilon}\right)
\end{aligned}
$$

Note that for any positive constant $\varepsilon>0$ and $\boldsymbol{v} \in \mathbb{R}^{n}$ the following inequality holds [60]:

$$
\|\boldsymbol{v}\|-\boldsymbol{v}^{T} \tanh \left(\frac{\boldsymbol{v}}{\varepsilon}\right) \leq k_{t} \varepsilon
$$

where $k_{t}=e^{-\left(k_{t}+1\right)}$, i.e. $k_{t}=0.2785$.

We further have

$$
\left\{\begin{array}{l}
L_{1}\left\|\boldsymbol{s}_{12}\right\| \leq L_{1}\left(\boldsymbol{s}_{12}^{T} \tanh \left(\frac{\boldsymbol{s}_{12}}{\varepsilon}\right)+k_{t} \varepsilon\right) \\
L_{i}\left\|\boldsymbol{s}_{i}\right\| \leq L_{i}\left(\boldsymbol{s}_{i}^{T} \tanh \left(\frac{\boldsymbol{s}_{i}}{\varepsilon}\right)+k_{t} \varepsilon\right), i=2,3,4,5 \\
\rho_{1}\left\|\boldsymbol{s}_{12}\right\| \leq \rho_{1}\left(\boldsymbol{s}_{12}^{T} \tanh \left(\frac{\boldsymbol{s}_{12}}{\varepsilon}\right)+k_{t} \varepsilon\right) \\
\rho_{3}\left\|\boldsymbol{s}_{3}\right\| \leq \rho_{3}\left(\boldsymbol{s}_{3}^{T} \tanh \left(\frac{\boldsymbol{s}_{3}}{\varepsilon}\right)+k_{t} \varepsilon\right) \\
\rho_{4}\left\|\boldsymbol{s}_{4}\right\| \leq \rho_{4}\left(\boldsymbol{s}_{4}^{T} \tanh \left(\frac{\boldsymbol{s}_{4}}{\varepsilon}\right)+k_{t} \varepsilon\right)
\end{array}\right.
$$


Substituting (118) into (116) yields

$$
\begin{aligned}
\dot{V} \leq & \sum_{i=2}^{5}\left[-\boldsymbol{s}_{i}^{T} \boldsymbol{P}_{i} \boldsymbol{s}_{i}+\left(\boldsymbol{s}_{i}^{T} \tanh \left(\frac{\boldsymbol{s}_{i}}{\varepsilon}\right)-\kappa_{L, i}^{-1} \dot{\hat{L}}_{i}\right) \tilde{L}_{i}\right. \\
& \left.+\beta_{i} \boldsymbol{s}_{i}^{T} \boldsymbol{y}_{i}+\boldsymbol{y}_{i}^{T} \dot{\boldsymbol{y}}_{i}+k_{t} \varepsilon L_{i}\right] \\
& +\left(\boldsymbol{s}_{12}^{T} \tanh \left(\frac{\boldsymbol{s}_{12}}{\varepsilon}\right)-\kappa_{\rho, 1}^{-1} \dot{\hat{\rho}}_{1}\right) \tilde{\rho}_{1}+k_{t} \varepsilon \rho_{1} \\
& +\left(\boldsymbol{s}_{3}^{T} \tanh \left(\frac{\boldsymbol{s}_{3}}{\varepsilon}\right)-\kappa_{\rho, 3}^{-1} \dot{\hat{\rho}}_{3}\right) \tilde{\rho}_{3}+k_{t} \varepsilon \rho_{3} \\
& +\left(\boldsymbol{s}_{4}^{T} \tanh \left(\frac{\boldsymbol{s}_{4}}{\varepsilon}\right)-\kappa_{\rho, 3}^{-1} \dot{\hat{\rho}}_{4}\right) \tilde{\rho}_{4}+k_{t} \varepsilon \rho_{4} \\
& -\boldsymbol{s}_{11}^{T} \boldsymbol{P}_{11} \boldsymbol{s}_{11}-\boldsymbol{s}_{12}^{T} \boldsymbol{P}_{12} \boldsymbol{s}_{12}+\boldsymbol{s}_{11}^{T} \boldsymbol{y}_{1}+\boldsymbol{s}_{2}^{T} \boldsymbol{G}_{2} \boldsymbol{y}_{3}+\boldsymbol{y}_{1}^{T} \dot{\boldsymbol{y}}_{1} \\
& +\left(\boldsymbol{s}_{12}^{T} \tanh \left(\frac{\boldsymbol{s}_{12}}{\varepsilon}\right)-\kappa_{\kappa, 1}^{-1} \dot{\hat{L}}_{1}\right) \tilde{L}_{1}+k_{t} \varepsilon L_{1}
\end{aligned}
$$

Applying (70), (80), (93), (106) and (113) to (119), we further have

$$
\begin{aligned}
\dot{V} \leq & \sum_{i=2}^{5}\left[-\boldsymbol{s}_{i}^{T} \boldsymbol{P}_{i} \boldsymbol{s}_{i}+\frac{\gamma_{i 1}}{\kappa_{L, i}} \hat{L}_{i} \tilde{L}_{i}+\beta_{i} \boldsymbol{s}_{i}^{T} \boldsymbol{y}_{i}+\boldsymbol{y}_{i}^{T} \dot{\boldsymbol{y}}_{i}+k_{t} \varepsilon L_{i}\right] \\
& +\frac{\gamma_{12}}{\kappa_{\rho, 1}} \hat{\rho}_{1} \tilde{\rho}_{1}+\frac{\gamma_{32}}{\kappa_{\rho, 3}} \hat{\rho}_{3} \tilde{\rho}_{3}+\frac{\gamma_{42}}{\kappa_{\rho, 4}} \hat{\rho}_{4} \tilde{\rho}_{4}+k_{t} \varepsilon \rho_{1}+k_{t} \varepsilon \rho_{3} \\
& +k_{t} \varepsilon \rho_{4}-\boldsymbol{s}_{11}^{T} \boldsymbol{P}_{11} \boldsymbol{s}_{11}+\boldsymbol{s}_{11}^{T} \boldsymbol{y}_{1}+\boldsymbol{s}_{2}^{T} \boldsymbol{G}_{2} \boldsymbol{y}_{3}-\boldsymbol{s}_{12}^{T} \boldsymbol{P}_{12} \boldsymbol{s}_{12} \\
& +\frac{\gamma_{11}}{\kappa_{L, 1}} \hat{L}_{1} \tilde{L}_{1}+\boldsymbol{y}_{1}^{T} \dot{y}_{1}+k_{t} \varepsilon L_{1}
\end{aligned}
$$

From (66), (75), (91), (101) and (108), we can obtain

$$
\left\{\begin{array}{l}
\dot{y}_{1}=-\frac{\boldsymbol{y}_{1}}{\epsilon_{1}}-\dot{\chi}_{12 d}+\frac{\boldsymbol{\mu}_{1}}{\epsilon_{1}}+\dot{\boldsymbol{\mu}}_{1} \\
\dot{\boldsymbol{y}}_{2}=-\frac{\boldsymbol{y}_{2}}{\epsilon_{2}}-\dot{\chi}_{2 d} \\
\dot{\boldsymbol{y}}_{i}=-\frac{\boldsymbol{y}_{i}}{\epsilon_{i}}-\dot{\boldsymbol{\chi}}_{i d}+\frac{\boldsymbol{\mu}_{i}}{\epsilon_{i}}+\dot{\boldsymbol{\mu}}_{i}, \quad i=3,4,5
\end{array}\right.
$$

In this context, we have

$$
\begin{aligned}
\left\|\dot{\boldsymbol{y}}_{1}+\boldsymbol{y}_{1} / \epsilon_{1}\right\| & \leq z_{1}\left(\dot{\boldsymbol{\chi}}_{12 d}, \dot{\boldsymbol{\mu}}_{1}, \boldsymbol{\mu}_{1}\right) \\
\left\|\dot{\boldsymbol{y}}_{2}+\boldsymbol{y}_{2} / \epsilon_{2}\right\| & \leq z_{2}\left(\boldsymbol{\chi}_{2 d}\right) \\
\left\|\dot{\boldsymbol{y}}_{3}+\boldsymbol{y}_{3} / \epsilon_{3}\right\| & \leq z_{3}\left(\dot{\boldsymbol{\chi}}_{3 d}, \dot{\boldsymbol{\mu}}_{3}, \boldsymbol{\mu}_{3}\right) \\
\left\|\dot{\boldsymbol{y}}_{4}+\boldsymbol{y}_{4} / \epsilon_{4}\right\| & \leq z_{4}\left(\dot{\boldsymbol{\chi}}_{4 d}, \dot{\boldsymbol{\mu}}_{4}, \boldsymbol{\mu}_{4}\right) \\
\left\|\dot{\boldsymbol{y}}_{5}+\boldsymbol{y}_{5} / \epsilon_{5}\right\| & \leq z_{5}\left(\dot{\boldsymbol{\chi}}_{5 d}, \dot{\boldsymbol{\mu}}_{5}, \boldsymbol{\mu}_{5}\right)
\end{aligned}
$$

for continuously bounded functions $z_{i}(\cdot)$.

Together with (122)-(126), we eventually have

$$
\boldsymbol{y}_{i}^{T} \dot{\boldsymbol{y}}_{i} \leq-\left(\frac{1}{\epsilon_{i}}-\frac{1}{2}\right) \boldsymbol{y}_{i}^{T} \boldsymbol{y}_{i}+\frac{1}{2} z_{i}^{2}, \quad i=1, \cdots, 5
$$

In addition, using the Young's inequality yields

$$
\left\{\begin{array}{l}
\hat{L}_{i} \tilde{L}_{i} \leq \frac{1}{2} L_{i}^{2}-\frac{1}{2} \tilde{L}_{i}^{2}, i=1, \cdots, 5 \\
\hat{\rho}_{1} \tilde{\rho}_{1} \leq \frac{1}{2} \rho_{1}^{2}-\frac{1}{2} \tilde{\rho}_{1}^{2} \\
\hat{\rho}_{3} \tilde{\rho}_{3} \leq \frac{1}{2} \rho_{3}^{2}-\frac{1}{2} \tilde{\rho}_{3}^{2} \\
\hat{\rho}_{4} \tilde{\rho}_{4} \leq \frac{1}{2} \rho_{4}^{2}-\frac{1}{2} \tilde{\rho}_{4}^{2}
\end{array}\right.
$$

Applying (127) and (128) to (120) yields

$$
\begin{aligned}
\dot{V} \leq & -\boldsymbol{s}_{11}^{T}\left(\boldsymbol{P}_{11}-\frac{\boldsymbol{I}_{1}}{2}\right) \boldsymbol{s}_{11}-\boldsymbol{s}_{12}^{T} \boldsymbol{P}_{12} \boldsymbol{s}_{12} \\
& -\boldsymbol{s}_{2}^{T}\left(\boldsymbol{P}_{2}-\frac{\left(\beta_{2}+1\right) \boldsymbol{I}_{2}}{2}\right) \boldsymbol{s}_{2}-\sum_{i=3}^{5} \boldsymbol{s}_{i}^{T}\left(\boldsymbol{P}_{i}-\frac{\beta_{i} \boldsymbol{I}_{i}}{2}\right) \boldsymbol{s}_{i}
\end{aligned}
$$

$$
\begin{aligned}
& -\left(\frac{1}{\epsilon_{1}}-1\right) \boldsymbol{y}_{1}^{T} \boldsymbol{y}_{1}-\left(\frac{1}{\epsilon_{3}}-\frac{2+\beta_{3}}{2}\right) \boldsymbol{y}_{3}^{T} \boldsymbol{y}_{3} \\
& -\sum_{i=2,4,5}\left(\frac{1}{\epsilon_{i}}-\frac{1+\beta_{i}}{2}\right) \boldsymbol{y}_{i}^{T} \boldsymbol{y}_{i} \\
& -\sum_{i=1}^{5} \frac{\gamma_{i 1}}{2 \kappa_{L, i}} \tilde{L}_{i}^{2}-\sum_{i=1,3,4} \frac{\gamma_{i 2}}{2 \kappa_{\rho, i}} \tilde{\rho}_{i}^{2}+\sum_{i=1}^{5}\left[\frac{\gamma_{i 1}}{2 \kappa_{L, i}} L_{i}^{2}+k_{t} \varepsilon L_{i}\right] \\
& +\sum_{i=1,3,4}\left[\frac{\gamma_{i 2}}{2 \kappa_{\rho, i}} \rho_{i}^{2}+k_{t} \varepsilon \rho_{i}\right]+\sum_{i=1}^{5} \frac{1}{2} z_{i}^{2}
\end{aligned}
$$

where $\boldsymbol{I}_{i}(i=1,2,3) \in \mathbb{R}^{3}$ and $\boldsymbol{I}_{i}(i=4,5) \in \mathbb{R}^{4}$ are unity matrixes.

Selecting user-defined parameters satisfying the following conditions:

$\boldsymbol{P}_{11} \geq \frac{1+\alpha}{2} \boldsymbol{I}_{1}, \boldsymbol{P}_{12} \geq \frac{\alpha}{2} \boldsymbol{I}_{1}, \boldsymbol{P}_{2} \geq \frac{\beta_{2}+\alpha+1}{2} \boldsymbol{I}_{2}$,

$\boldsymbol{P}_{i} \geq \frac{\beta_{i}+\alpha}{2} \boldsymbol{I}_{i}, i=3,4,5$

$\frac{1}{\epsilon_{1}} \geq 1+\frac{\alpha}{2}, \frac{1}{\epsilon_{3}} \geq 1+\frac{\beta_{3}+\alpha}{2}, \frac{1}{\epsilon_{i}} \geq \frac{1+\beta_{i}+\alpha}{2}, i=2,4,5$

$\frac{\gamma_{i 1}}{\kappa_{L, i}} \geq \alpha, i=1, \cdots, 5, \frac{\gamma_{i 2}}{2 \kappa_{\rho, i}} \geq \alpha, i=1,3,4$

where $\alpha>0$ is any positive constant, we have

$$
\dot{V} \leq-\alpha V+C
$$

with

$$
\begin{aligned}
C= & \sum_{i=1}^{5}\left[\frac{\gamma_{i 1}}{2 \kappa_{L, i}} L_{i}^{2}+k_{t} \varepsilon L_{i}\right] \\
& +\sum_{i=1,3,4}\left[\frac{\gamma_{i 2}}{2 \kappa_{\rho, i}} \rho_{i}^{2}+k_{t} \varepsilon \rho_{i}\right]+\sum_{i=1}^{5} \frac{1}{2} \bar{z}_{i}^{2}
\end{aligned}
$$

where $\bar{z}_{i}$ is the upper bound of function $z_{i}$.

Together with (115) and (131), we have

$$
0 \leq V(t) \leq V(0) e^{-\alpha t}+\left(1-e^{-\alpha t}\right) \frac{C}{\alpha}<\infty
$$

It is clear that $V(t)$ is bounded. Moreover, together with (115), there exist a finite time $T>0$ such that

$$
\begin{aligned}
& V= \frac{1}{2}\left[\sum_{i=2}^{5} \boldsymbol{s}_{i}^{T} \boldsymbol{s}_{i}+\boldsymbol{s}_{11}^{T} \boldsymbol{s}_{11}+\boldsymbol{s}_{12}^{T} \boldsymbol{s}_{12}+\sum_{i=1}^{5} \boldsymbol{y}_{i}^{T} \boldsymbol{y}_{i}\right. \\
&\left.+\sum_{i=1}^{5} \kappa_{L, i}^{-1} \tilde{L}_{i}^{2}+\kappa_{\rho, 1}^{-1} \tilde{\rho}_{1}^{2}+\kappa_{\rho, 3}^{-1} \tilde{\rho}_{3}^{2}+\kappa_{\rho, 4}^{-1} \tilde{\rho}_{4}^{2}\right] \\
& \leq 2 C / \alpha, \quad \forall t \geq T
\end{aligned}
$$

In this context, we have

$$
\vartheta \leq 2 \sqrt{C / \alpha}
$$

where $\vartheta \in\left\{\left\|s_{11}\right\|,\left\|s_{12}\right\|,\left\|s_{2}\right\|, \cdots,\left\|s_{5}\right\|,\left\|y_{1}\right\|, \cdots,\left\|y_{5}\right\|\right.$, $\left.\left|\tilde{L}_{1}\right|, \cdots,\left|\tilde{L}_{5}\right|,\left|\tilde{\rho}_{1}\right|,\left|\tilde{\rho}_{3}\right|,\left|\tilde{\rho}_{4}\right|\right\}$.

Using (134) and Lemma 1, we immediately have the tracking error $\left\|\boldsymbol{e}_{11}\right\|$ is uniformly bounded. Similarly, we can obtain that all the other signals including $\boldsymbol{e}_{12}, \boldsymbol{e}_{2}, \cdots, \boldsymbol{e}_{5}$, $\boldsymbol{y}_{1}, \cdots, \boldsymbol{y}_{5}, \tilde{L}_{1}, \cdots, \tilde{L}_{5}, \tilde{\rho}_{1}, \tilde{\rho}_{3}$ and $\tilde{\rho}_{4}$ are ultimately uniformly bounded. Together with the filtered dynamics (66), (75), (91), 
TABLE I

MAIN PARAMETERS OF THE QUAV.

\begin{tabular}{l|l|l||l|l|l}
\hline Para. & Value & Units & Para. & Value & Units \\
\hline \hline$g$ & 9.806 & $\mathrm{~m} / \mathrm{s}^{2}$ & $\mathrm{~m}$ & 0.65 & $\mathrm{~kg}$ \\
$b$ & $7.5 \mathrm{e}-7$ & - & $l$ & 0.232 & $\mathrm{~m}$ \\
$c$ & $1 \mathrm{e}-5$ & - & $n$ & $1.5 \mathrm{e}-4$ & - \\
$k$ & $3.13 \mathrm{e}-5$ & - & $r_{s}$ & 1 & - \\
$C_{m}$ & 0.08 & - & $C_{e}$ & 0.0415 & - \\
$J_{m}$ & $4 \mathrm{e}-4$ & $N /(\mathrm{m} / s)^{2}$ & $J_{r}$ & $6 \mathrm{e}-3$ & $\mathrm{~N} /(\mathrm{m} / \mathrm{s})^{2}$ \\
$R_{a}$ & 0.036 & $\Omega$ & $I_{r}$ & $1 \mathrm{e}-3$ & $\mathrm{~kg} \cdot \mathrm{m}^{2}$ \\
$D_{x}$ & $1 \mathrm{e}-6$ & $N /(\mathrm{m} / \mathrm{s})^{2}$ & $J_{x}$ & 0.015 & $\mathrm{~kg} \cdot \mathrm{m}^{2}$ \\
$D_{y}$ & $1 \mathrm{e}-6$ & $N /(\mathrm{m} / \mathrm{s})^{2}$ & $J_{y}$ & 0.015 & $\mathrm{~kg} \cdot \mathrm{m}^{2}$ \\
$D_{z}$ & $1 \mathrm{e}-4$ & $N /(\mathrm{m} / \mathrm{s})^{2}$ & $J_{z}$ & 0.026 & $\mathrm{~kg} \cdot \mathrm{m}^{2}$ \\
$w_{d}^{m}$ & 3000 & $r p m$ & $w_{e d}^{m}$ & 3000 & $\mathrm{rpm}$ \\
$u_{5}^{0}$ & 0.2 & $V$ & $u_{5}^{m}$ & 14 & $\mathrm{~V}$ \\
$\eta$ & 0.9 & - & & & \\
\hline
\end{tabular}

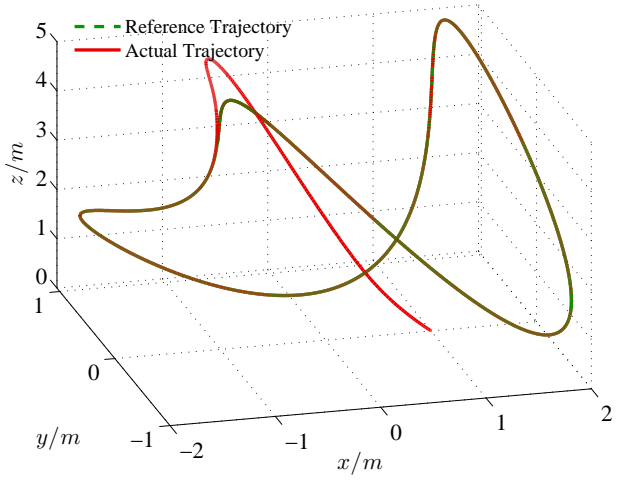

Fig. 5. Trajectory tracking of the BCTTC scheme.
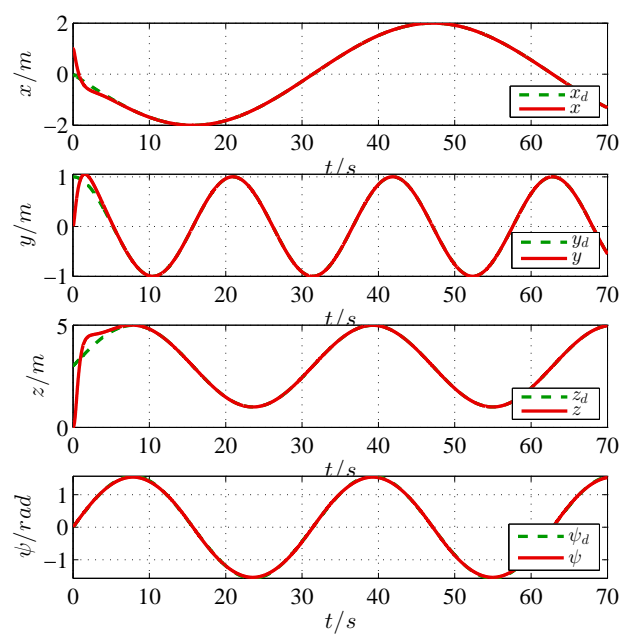

Fig. 6. Desired and actual states $x, y, z$ and $\psi$.

(101) and (108), and the UAC mechanism (70), (80), (93), (106) and (113), we can obtain that system signals including $\dot{\bar{\chi}}_{12 d}, \dot{\bar{\chi}}_{2 d}, \cdots, \dot{\bar{\chi}}_{5 d}, \dot{\boldsymbol{y}}_{1}, \cdots, \dot{\boldsymbol{y}}_{5}, \dot{\hat{L}}_{1}, \cdots, \dot{\hat{L}}_{5}, \dot{\hat{\rho}}_{1}, \dot{\hat{\rho}}_{3}$ and $\dot{\hat{\rho}}_{4}$ are bounded. This concludes the proof.

\section{Simulation STUdies}

In this section, the effectiveness and superiority of the proposed BCTTC scheme is demonstrated for trajectory tracking control of a complex QUAV with actuator dynamics and cascade constraints on both control input and states, in the
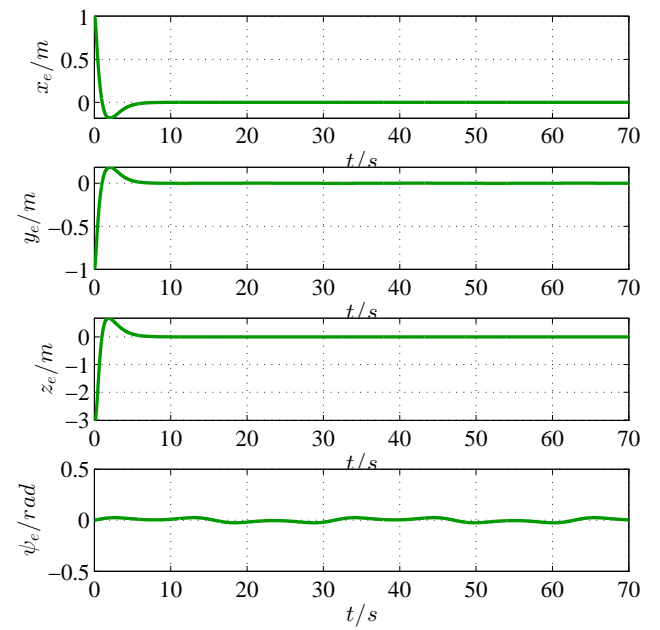

Fig. 7. Trajectory tracking errors $x_{e}, y_{e}, z_{e}$ and $\psi_{e}$.
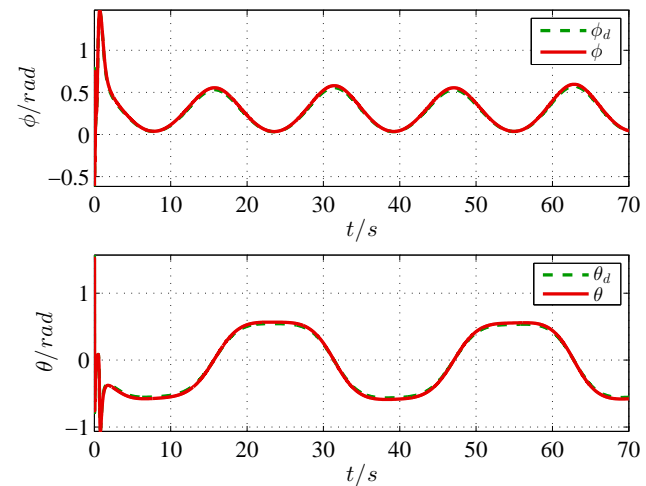

Fig. 8. Desired and actual Euler angles $\phi$ and $\theta$.
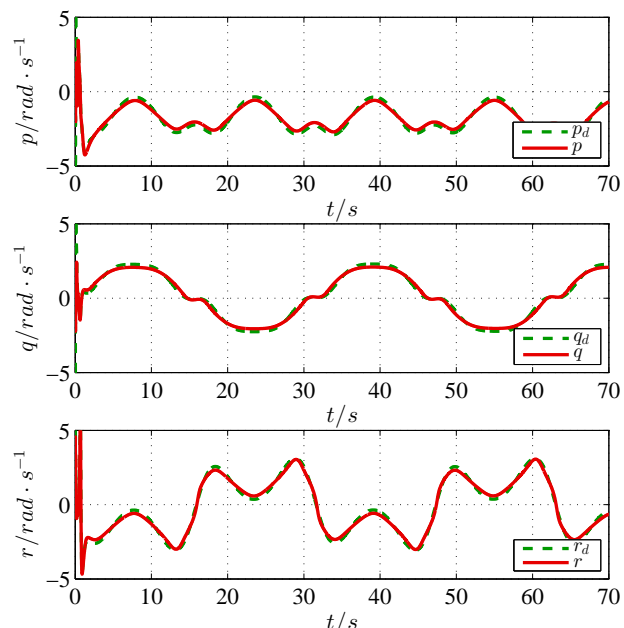

Fig. 9. Desired and actual angular velocities $p, q$ and $r$.

presence of complex unknowns. Main parameters of the QUAV refer to [58] and are listed in Table I.

The reference trajectory is governed by $x_{d}=-2 \sin (0.1 t)$, $y_{d}=\cos (0.3 t), z_{d}=2 \sin (0.2 t)+3$ and $\psi_{d}=\frac{\pi}{2} \sin (0.2 t)$, and the initial condition is as follows: $\chi_{11}(0)=[1,0,0]^{T}$, $\chi_{12}(0)=[0,0,0]^{T}$ and $\chi_{2}(0)=[0,0,0]^{T}$. For the sake of simulation studies, complex unknowns are assumed to be as follows: $\boldsymbol{d}_{1}=5\left[\sin \left(0.01 \chi_{11}^{T} \chi_{12}\right), \cos \left(0.02 \chi_{11}^{T} \chi_{12}\right)\right.$, $\left.\sin \left(0.05 \chi_{11}^{T} \chi_{12}\right) \cos \left(0.03 \chi_{11}^{T} \chi_{12}\right)\right]^{T}, \boldsymbol{d}_{2}=5 \sin \left(\boldsymbol{\chi}_{2}\right) \cos \left(\boldsymbol{\chi}_{2}\right)$, $\boldsymbol{d}_{3}=5 \sin \left(\chi_{3}\right) \cos \left(\chi_{3}\right), \boldsymbol{d}_{4}=100 \sin ^{2}\left(\chi_{4}\right) \cos \left(\chi_{4}\right)$, and 

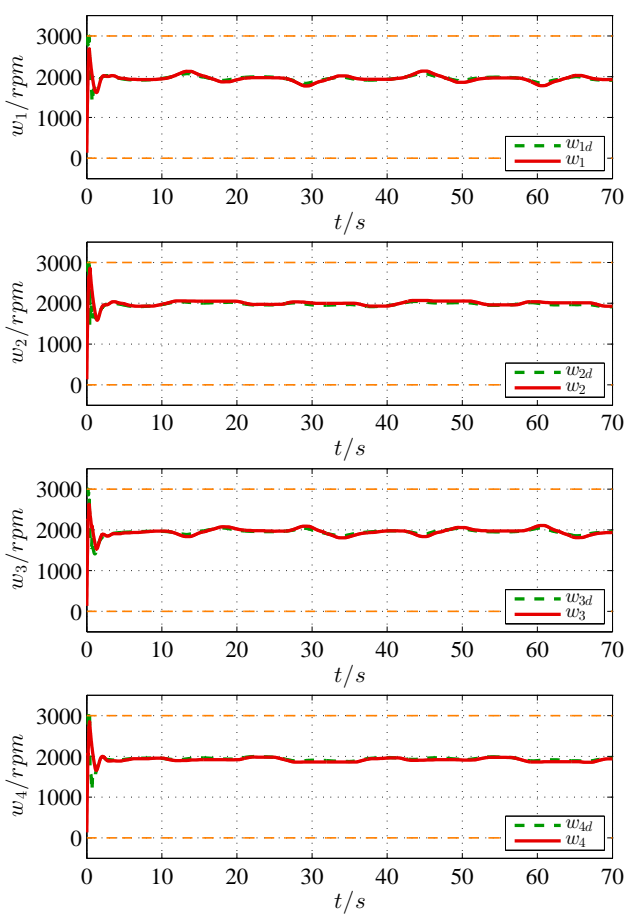

Fig. 10. Desired and actual propeller speeds $w_{1}, w_{\rho}, w_{3}$ and $w_{4}$.
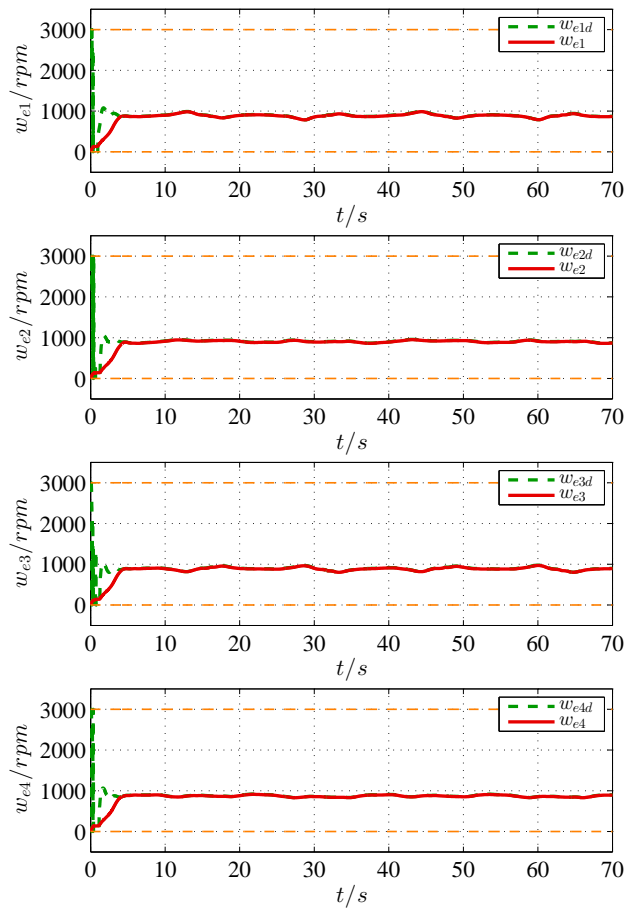

Fig. 11. Desired and actual motor rotations $w_{e 1}, w_{e 2}, w_{e 3}$ and $w_{e 4}$.

$\boldsymbol{d}_{5}=100 \cos \left(0.01 \chi_{5}\right)$.

User-defined parameters of the BCTTC scheme are as follows: $\boldsymbol{P}_{11}=\boldsymbol{P}_{12}=\boldsymbol{P}_{3}=\operatorname{diag}(10,10,10)$, $\boldsymbol{P}_{2}=\operatorname{diag}(100,100,100), \quad \boldsymbol{P}_{4}=\operatorname{diag}(1,1,1,1)$, $\boldsymbol{P}_{5}=\operatorname{diag}(10,10,10,10), \boldsymbol{K}_{11}=\boldsymbol{K}_{12}=$ $\boldsymbol{K}_{2}=\operatorname{diag}(0.01,0.01,0.01), \quad \boldsymbol{K}_{4}=\boldsymbol{K}_{5}=$ $\operatorname{diag}(0.01,0.01,0.01,0.01), \gamma_{11}=\gamma_{21}=\gamma_{31}=\gamma_{41}=$ $\gamma_{51}=1, \gamma_{12}=\gamma_{32}=\gamma_{42}=2, \kappa_{L, 1}=\kappa_{L, 2}=\kappa_{L, 3}=$
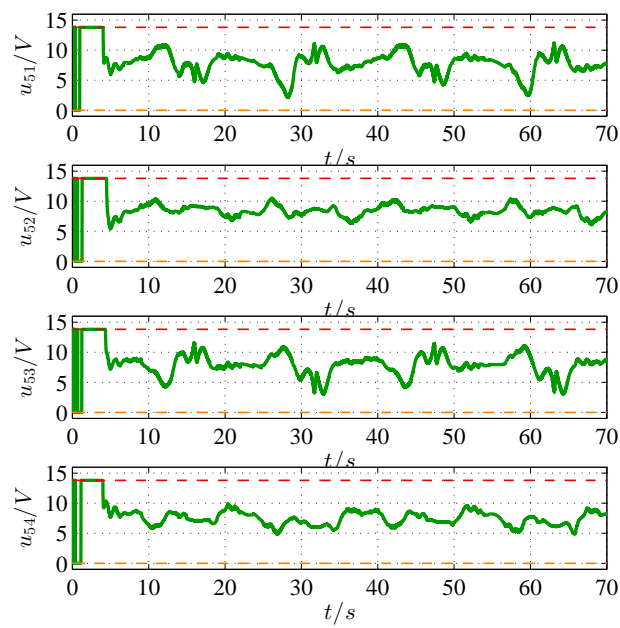

Fig. 12. Control inputs.

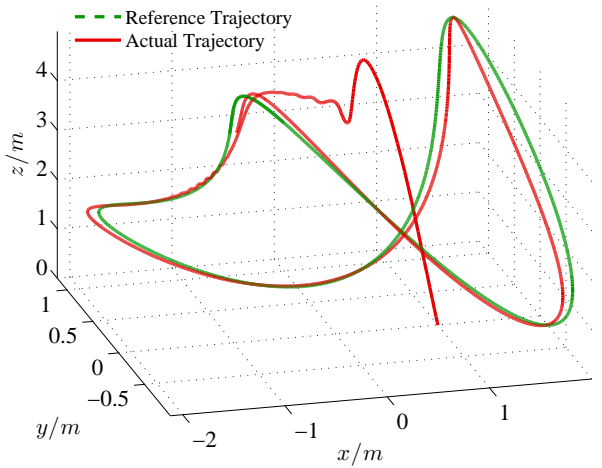

Fig. 13. Trajectory tracking of PD control scheme.
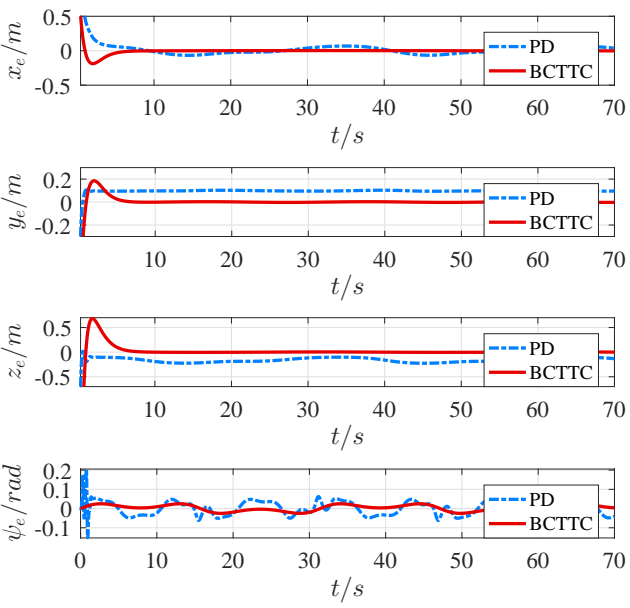

Fig. 14. Comparisons of trajectory tracking errors.

$\kappa_{L, 4}=\kappa_{L, 5}=1, \kappa_{\rho, 1}=\kappa_{\rho, 3}=\kappa_{\rho, 4}=2, \varepsilon=1$, $\epsilon_{1}=\epsilon_{2}=\epsilon_{3}=\epsilon_{4}=\epsilon_{5}=0.01$, and $\beta_{2}=\beta_{3}=\beta_{4}=\beta_{5}=1$.

The actual and reference trajectories in $3-\mathrm{D}$ space are shown in Fig. 5, from which we can see that the BCTTC scheme can render the QUAV track the desired trajectory accurately in the presence of both mismatched and matched complex unknowns. Individual positions, i.e., $x, y$ and $z$, and the yaw angle $\psi$ together with their desired targets are shown in Fig. 6, from which we can see that the QUAV using the BCTTC scheme can track the desired individual trajectories with fast response 
TABLE II

Performance Comparisons of BCTTC With PD.

\begin{tabular}{c|c|c|c|c}
\hline \multirow{2}{*}{} & \multicolumn{2}{|c|}{ BCTTC } & \multicolumn{2}{c}{ PD } \\
\cline { 2 - 5 } & IAE & ITAE & IAE & ITAE \\
\hline \hline$x_{e}$ & 0.9773 & $0.0187 \mathrm{E}+4$ & 3.8597 & $0.9520 \mathrm{E}+4$ \\
\hline$y_{e}$ & 0.9726 & $0.0291 \mathrm{E}+4$ & 3.0425 & $1.0152 \mathrm{E}+4$ \\
\hline$z_{e}$ & 1.3784 & $0.0620 \mathrm{E}+4$ & 2.7317 & $0.8251 \mathrm{E}+4$ \\
\hline$\psi_{e}$ & 0.9928 & $0.3509 \mathrm{E}+4$ & 2.1992 & $0.7405 \mathrm{E}+4$ \\
\hline
\end{tabular}
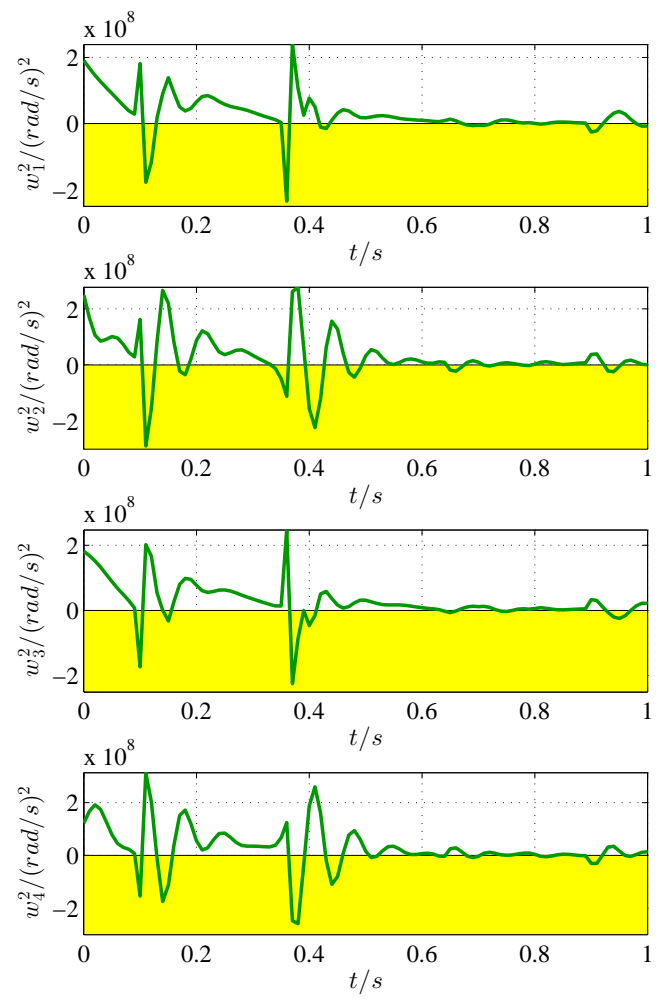

Fig. 15. Rotation squares of PD control scheme.

and high accuracy, simultaneously, whereby tracking errors are shown in Fig. 7. Intermediate tracking results for other states including Euler angles, angular velocities, propeller speeds and motor rotations are shown Figs. 8-11, respectively, which demonstrate that accurate tracking of intermediate states can still be guaranteed under the constraints on propeller speeds and motor rotations (shown in Figs. 10 and 11). Eventually, control inputs to 4 motors are shown in Fig. 12, from which we can see that nonreversible constraints and saturations have been effectively addressed.

Furthermore, in order to demonstrate the superiority of the proposed BCTTC scheme, comprehensive comparisons with a PD control scheme are conducted on previous settings. To this end, PD controllers are designed as follows:

$$
\begin{aligned}
U_{1} & =K_{p 1}\left(\chi_{11}-\chi_{11 d}\right)+K_{d 1}\left(\chi_{12}-\chi_{12 d}\right) \\
U_{2} & =K_{p 2}\left(\phi-\phi_{d}\right)+K_{d 2}\left(\dot{\phi}-\dot{\phi}_{d}\right) \\
U_{3} & =K_{p 3}\left(\theta-\theta_{d}\right)+K_{d 3}\left(\dot{\theta}-\dot{\theta}_{d}\right) \\
U_{4} & =K_{p 4}\left(\psi-\psi_{d}\right)+K_{d 4}\left(\dot{\psi}-\dot{\psi}_{d}\right)
\end{aligned}
$$

where $U_{1}$ is control input of position dynamics (4), $U_{2}:=$ $l b\left(-w_{2}^{2}+w_{4}^{2}\right), U_{3}:=l b\left(-w_{1}^{2}+w_{3}^{2}\right)$ and $U_{4}:=k\left(-w_{1}^{2}+\right.$ $\left.w_{2}^{2}-w_{3}^{2}+w_{4}^{2}\right)$ are control inputs of attitude dynamics (11), $\phi_{d}, \theta_{d}$ and $T:=m\left\|\boldsymbol{U}_{1}\right\|$ are derived from $\boldsymbol{U}_{1}$ according to (74), and fine-tuning parameters are chosen as follows: $\boldsymbol{K}_{p 1}=$ $\operatorname{diag}(20,100,150), \boldsymbol{K}_{d 1}=\operatorname{diag}(10,10,10)$, and $K_{p 2}=1$, $K_{d 2}=0.3, K_{p 3}=1, K_{d 3}=0.3, K_{p 4}=1.5$, and $K_{d 4}=0.5$.

Trajectory tracking result of the PD control approach and comparisons with the BCTTC scheme are shown in Figs. 13 and 14, respectively, and illustrate that the BCTTC approach can accommodate complex unknowns, and thereby achieving nearly zero steady-state discrepancies which apparently appear in PD controllers.

In order to make intensive insight into the superiority of the BCTTC, quantitative comparisons using Integrated Absolute Error (IAE) and Integrated Time Absolute Error (ITAE) indices for tracking errors are summarized in Table II. Clearly, it can be seen that the proposed BCTTC scheme is significantly superior to the PD control approach. It should be noted that the PD control strategy cannot tackle constrained actuator dynamics. As a consequence, as shown in Fig. 15, negative squares of rotor rotations reversely deriving from PD control input torques would unreasonably occur, and thereby leading to unreachable control efforts in practice and even destroying system stability. Similarly, those methods taking rotor torques as control inputs would inevitably suffer from the aforementioned negative-square dilemma. In this context, the proposed BCTTC scheme via backpropagating constraints due to constrained actuator dynamics can definitely guarantee reasonable control signals which can be completely executed by actuators.

\section{CONCLUSIONS}

In this paper, the BCTTC scheme for trajectory tracking of a QUAV with constrained actuator dynamics and complex unknowns has been proposed. Unlike previous works, the entire QUAV system has been decomposed into 5 cascade subsystems connected by intermediate nonlinearities. In this context, SMC-based sub-controllers have been recursively designed by addressing underactuation and cascade constraints, whereby the preceding sub-controller provides desired signals for the succeeding subsystem. In addition, first-order filters have been employed to avoid the smoothness requirement and decouple the iterative design within the backsteppinglike procedure. By virtue of backpropagating constraints (BC), intermediate controls have been shaped within reachable regions determined by constrained actuator dynamics including saturations and dead zones. Furthermore, universal adaptive compensators have been employed to dominate complex unknowns together with BC-based intermediate discrepancies. Using the Lyapunov approach, BCTTC tracking errors can be made arbitrarily small and all signals are bounded. Simulation studies have shown that the proposed BCTTC scheme can achieve high-accuracy tracking under constrained actuator dynamics and complex unknowns, and is remarkably superior to previous approaches without addressing actuator constraints or inner nonlinearities. 


\section{ACKNOWLEDGMENT}

The authors would like to thank Editor-in-Chief, Associate Editor and anonymous referees for their invaluable comments and suggestions.

\section{REFERENCES}

[1] A. Tayebi and S. McGilvray, "Attitude stabilization of a VTOL quadrotor aircraft," IEEE Trans. Control Syst. Technol., vol. 14, no. 3, pp. 562-571, May 2006.

[2] A. Roberts and A. Tayebi, "Adaptive position tracking of VTOL UAVs," IEEE Trans. Robot., vol. 27, no. 1, pp. 129-142, Feb. 2011.

[3] A. Abdessameud and A. Tayebi, "Global trajectory tracking control of VTOL-UAVs without linear velocity measurements," Automatica, vol. 46, no. 6, pp. 1053-1059, Jun. 2010.

[4] K. D. Do, "Coordination control of quadrotor VTOL aircraft in threedimensional space," Int. J. Control, vol. 88, no. 3, pp. 543-558, Mar. 2015.

[5] W. He, Z. Yan, C. Sun, and Y. Chen, "Adaptive neural network control of a flapping wing micro aerial vehicle with disturbance observer," IEEE Trans. Cybern., vol. 47, no. 10, pp. 3452-3465, Oct. 2017.

[6] W. He and S. Zhang, "Control design for nonlinear flexible wings of a robotic aircraft," IEEE Trans. Control Syst. Technol., vol. 25, no. 1, pp. 351-357, Jan. 2017.

[7] D. Cabecinhas, R. Naldi, C. Silvestre, R. Cunha, and L. Marconi, "Robust landing and sliding maneuver hybrid controller for a quadrotor vehicle," IEEE Trans. Control Syst. Technol., vol. 24, no. 2, pp. 400-412, Mar. 2016.

[8] S. Driessens and P. Pounds, "The triangular quadrotor: A more efficient quadrotor configuration," IEEE Trans. Robot. Autom., vol. 31, no. 6, pp. 1517-1526, Dec. 2015.

[9] M. Elfeky and M. Elshafei, "Quadrotor with tiltable totors for manned applications," in Proc. 11th Int. Multi. Conf. Syst. Signals Devices, Barcelona, Spain, 2014, pp. 1-5.

[10] K. Alexis, G. Nikolakopoulos, and A. Tzes, "Model predictive quadrotor control: Attitude, altitude and position experimental studies," IET Control Theory Appl., vol. 6, no. 12, pp. 1812-1827, Aug. 2012.

[11] A. Levant, "Higher-order sliding modes, differentiation and outputfeedback control," Int. J. Control, vol. 76, no. 9-10, pp. 924-941, Sep. 2003.

[12] Y. Wang, Y. Xia, H. Shen, and P. Zhou, "SMC design for robust stabilization of nonlinear Markovian jump singular systems," IEEE Trans. Autom. Control, vol. 63, no. 1, pp. 219-224, Jan. 2018.

[13] Y. Wang, H. Shen, H. R. Karimi, and D. Duan, "Dissipativity-based fuzzy integral sliding mode control of continuous-time T-S fuzzy systems," IEEE Trans. Fuzzy Syst., 2017, DOI: 10.1109/TFUZZ.2017.2710952.

[14] D. Swaroop, J. K. Hedrick, P. P. Yip, and J. C. Gerdes, "Dynamic surface control for a class of nonlinear systems," IEEE Trans. Autom. Control, vol. 45 , no. 10 , pp. $1893-1899$, Oct. 2000.

[15] N. Wang, J. C. Sun, and M. J. Er, "Tracking-error-based universal adaptive fuzzy control for output tracking of nonlinear systems with completely unknown dynamics," IEEE Trans. Fuzzy Syst., vol. 26, no. 2, pp. 869-883, Apr. 2018.

[16] N. Wang and M. J. Er, "Self-constructing adaptive robust fuzzy neural tracking control of surface vehicles with uncertainties and unknown disturbances," IEEE Trans. Control Syst. Technol., vol. 23, no. 3, pp. 991-1002, May 2015.

[17] N. Wang, M. J. Er, J. C. Sun, and Y. C. Liu, "Adaptive robust online constructive fuzzy control of a complex surface vehicle system," IEEE Trans. Cybern., vol. 46, no. 7, pp. 1511-1523, Jul. 2016.

[18] W. He, Y. Chen, and Z. Yin, "Adaptive neural network control of an uncertain robot with full-state constraints," IEEE Trans. Cybern., vol. 46, no. 3, pp. 620-629, Apr. 2016.

[19] W. He, Y. Dong, and C. Sun, "Adaptive neural impedance control of a robotic manipulator with input saturation," IEEE Trans. Syst., Man, Cybern., Syst., vol. 46, no. 3, pp. 334-344, Mar. 2016.

[20] Q. Zhou, H. Li, L. Wang, and R. Lu, "Prescribed performance observer-based adaptive fuzzy control for nonstrict-feedback stochastic nonlinear systems," IEEE Trans. Syst., Man, Cybern., Syst., 2017, DOI:10.1109/TSMC.2017.2738155.

[21] L. Wang, M. Basin, H. Li, and R. Lu, "Observer-based composite adaptive fuzzy control for nonstrict-feedback systems with actuator failures," IEEE Trans. Fuzzy Syst., 2017, DOI: 10.1109/TFUZZ.2017.2774185.
[22] N. Wang, S. F. Su, J. Yin, Z. Zheng, and M. J. Er, "Global asymptotic model-free trajectory-independent tracking control of an uncertain marine vehicle: An adaptive universe-based fuzzy control approach," IEEE Trans. Fuzzy Syst., 2017, DOI: 10.1109/TFUZZ.2017.2737405.

[23] W. He and Y. Dong, "Adaptive fuzzy neural network control for a constrained robot using impedance learning," IEEE Trans. Neural Netw. Learn. Syst., vol. 29, no. 4, pp. 1174-1186, Apr. 2018.

[24] N. Wang, J. C. Sun, M. Han, Z. Zheng, and M. J. Er, "Adaptive approximation-based regulation control for a class of uncertain nonlinear systems without feedback linearizability," IEEE Trans. Neural Netw. Learn. Syst., 2017, DOI: 10.1109/TNNLS.2017.2738918.

[25] N. Wang, L. S., W. Zhang, Z. Liu, and M. J. Er, "Finite-time observer based accurate tracking control of a marine vehicle with complex unknowns," Ocean Eng., vol. 145, pp. 406-415, 2017.

[26] N. Wang, C. Qian, and Z. Y. Sun, "Global asymptotic output tracking of nonlinear second-order systems with power integrators," Automatica, vol. 80, pp. 156-161, 2017.

[27] H. Voos, "Nonlinear control of a quadrotor micro-UAV using feedbacklinearization," in Proc. 5th IEEE Int. Conf. Mechatronics, Malaga, Spain, 2009, pp. 1-6.

[28] F. Y. Chen, R. Q. Jiang, K. K. Zhang, B. Jiang, and G. Tao, "Robust backstepping sliding mode control and observer-based fault estimation for a quadrotor UAV," IEEE Trans. Ind. Electron., vol. 63, no. 8, pp. 5044-5056, Aug. 2016.

[29] R. Xu and U. Özgüner, "Sliding mode control of a quadrotor helicopter," in Proc. IEEE Conf. Decision Control, San Diego, USA, 2006, pp. 49574962.

[30] D. M. Paul and L. Boris, "Position trajectory tracking of a quadrotor helicopter based on L1 adaptive control," in Proc. European Control Conf., Strasbourg, France, 2013, pp. 3346-3353.

[31] G. V. Raffo, M. G. Ortega, and F. R. Rubio, "An integral predictive/nonlinear $H_{\infty}$ control structure for a quadrotor helicopter," Automatica, vol. 46, no. 1, pp. 29-39, Jan. 2010.

[32] S. Islam, P. X. Liu, and A. E. Saddik, "Robust control of four-rotor unmanned aerial vehicle with disturbance uncertainty," IEEE Trans. Ind. Electron., vol. 62, no. 3, pp. 1563-1571, Mar. 2015.

[33] S. Bouabdallah, A. Noth, and R. Siegwart, "PID vs LQ control techniques applied to an indoor micro quadrotor," in Proc. IEEE/RSJ Int. Conf. Intell. Robot. Syst., Sendai, Japan, 2004, pp. 2451-2456.

[34] G. Szafranski and R. Czyba, "Different approaches of PID control UAV type quadrotor," in Proc. Int. Micro Air Veh. Conf. Comp., Gelderland, Netherlands, 2011, pp. 70-75.

[35] J. Zhu, E. Liu, S. Guo, and C. Xu, "A gradient optimization based PID tuning approach on quadrotor," in Proc. 27th Chinese Control Decision Conf., Qingdao, China, 2015, pp. 1588-1593.

[36] T. Dierks and S. Jagannathan, "Output feedback control of a quadrotor UAV using neural networks," IEEE Trans. Neural Netw., vol. 21, no. 1, pp. 50-66, Jan. 2010.

[37] E. N. Sanchez, H. M. Becerra, and C. M. Velez, "Combining fuzzy, PID and regulation control for an autonomous mini-helicopter," Inform. Sci., vol. 177, no. 10, pp. 1999-2022, May 2007.

[38] J. P. Ortiz, L. I. Minchala, and M. J. Reinoso, "Nonlinear robust HInfinity PID controller for the multivariable system quadrotor," IEEE Latin America Trans., vol. 14, no. 3, pp. 1176-1183, Mar. 2016.

[39] F. Chen, W. Lei, K. Zhang, G. Tao, and B. Jiang, "A novel nonlinear resilient control for a quadrotor UAV via backstepping control and nonlinear disturbance observer," Nonlinear Dyn., vol. 85, no. 2, pp. 1281-1295, Jul. 2016

[40] N. Wang, Z. Sun, J. Yin, S.-F. Su, and S. Sharma, "Finite-time observer based guidance and control of underactuated surface vehicles with unknown sideslip angles and disturbances," IEEE Access, vol. 6, pp. 14059-14 070, Jan. 2018.

[41] A. Das and F. Lewis, "Neural network-based robust backstepping control approach for quadrotors," in Proc. AIAA Guid. Navig. Control Conf. Exhib., Honolulu, Hawaii, 2008, pp. 1-17.

[42] T. Hamel, R. Mahony, R. Lozano, and J. Ostrowski, "Dynamic modeling and configuration stabilization for an X4-flyer," in Proc. IFAC 15th Triennial World Cong., Barcelona, Spain, 2002, pp. 217-222.

[43] S. Bouabdallah and R. Siegwart, "Full control of a quadrotor," in Proc. IEEE/RSJ Int. Conf. Intell. Robot. Syst., San Diego, USA, 2007, pp. $153-158$.

[44] A. Roza and M. Maggiore, "A class of position controllers for underactuated VTOL vehicles," IEEE Trans. Autom. Control, vol. 59, no. 9, pp. 2580-2585, Sep. 2014.

[45] N. Cao and A. F. Lynch, "Inner-outer loop control for quadrotor UAVs with input and state constraints," IEEE Trans. Control Syst. Technol., vol. 24, no. 5, pp. 1797-1804, Sep. 2016. 
[46] K. D. Do, Z. P. Jiang, and J. Pan, "On global tracking control of a VTOL aircraft without velocity measurements," IEEE Trans. Autom. Control, vol. 48, no. 12, pp. 2212-2217, Dec. 2003.

[47] T. Madani and A. Benallegue, "Backstepping control for a quadrotor helicopter," in Proc. IEEE/RSJ Int. Conf. Intell. Robot. Syst., Beijing, China, 2006, pp. 3255-3260.

[48] M. Huang, B. Xian, C. Diao, K. Yang, and Y. Feng, "Adaptive tracking control of underactuated quadrotor unmanned aerial vehicles via backstepping," in Proc. Amer. Control Conf., Baltimore, USA, 2010, pp. 2076-2081.

[49] J. Xiong and E. Zheng, "Position and attitude tracking control for a quadrotor UAV," ISA Trans., vol. 53, no. 3, pp. 725-731, May 2014.

[50] L. Besnard, Y. B. Shtessel, and B. Landrum, "Control of a quadrotor vehicle using sliding mode disturbance observer," in Proc. American Control Conf., New York City, USA, 2007, pp. 5230-5235.

[51] L. Besnard, Y. B. Shtesscl, and B. Landrum, "Quadrotor vehicle control via sliding mode control driven by sliding mode disturbance observer," J. Franklin Inst., vol. 349, no. 2, pp. 658-684, Mar. 2012.

[52] K. D. Do, Z. P. Jiang, and J. Pan, "Underactuated ship global tracking under relaxed conditions," IEEE Trans. Autom. Control, vol. 47, no. 9, pp. 1529-1536, Sep. 2002.

[53] S. Islam, J. Dias, and L. D. Seneviratne, "Adaptive tracking control for quadrotor unmanned flying vehicle," in Proc. IEEE/ASME Int. Conf. Adv. Intell. Mechatronics, Besancon, France, 2014, pp. 441-445.

[54] F. Yacef, O. Bouhali, M. Hamerlain, and N. Rizoug, "Observer-based adaptive fuzzy backstepping tracking control of quadrotor unmanned aerial vehicle powered by Li-ion battery," J. Intell. Robot. Syst., vol. 84, no. 1-4, pp. 179-197, Dec. 2016.

[55] P. Pounds, R. Mahony, and P. Corke, "Modelling and control of a large quadrotor robot," Control Eng. Pract., vol. 18, no. 7, pp. 725-731, Jul. 2010

[56] F. Sharifi, M. Mirzaei, B. W. Gordon, and Y. Zhang, "Fault tolerant control of a quadrotor UAV using sliding mode control," in Proc. Conf. Control Fault Tolerant Syst., Nice, France, 2010, pp. 239-244.

[57] A. U. Awan, "Adaptive tracking control of underactuated quadrotor unmanned aerial vehicles via backstepping," in Proc. 11th Int. Bhurban Conf. Appl. Sci. Technol., Islamabad, Pakistan, 2014, pp. 97-102.

[58] A. S. Sanca, P. J. Alsina, and J. F. Cerqueira, "Dynamic modelling of a quadrotor aerial vehicle with nonlinear inputs," in Proc. IEEE Latin Amer. Robot. Symp., Salvador, Brazil, 2008, pp. 143-148.

[59] H. Khalil, Nonlinear Systems, 2nd ed. Upper Saddle River, NJ: PrenticeHall, 1996.

[60] M. M. Polycarpou, "Stable adaptive neural control scheme for nonlinear systems," IEEE Trans. Autom. Control, vol. 41, no. 3, pp. 5044-5056, Mar. 1996.

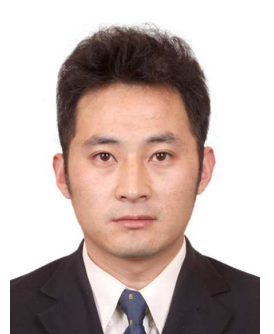

Ning Wang (S'08-M'12-SM'15) received his B. Eng. degree in Marine Engineering and the Ph.D. degree in control theory and engineering from the Dalian Maritime University (DMU), Dalian, China in 2004 and 2009, respectively. From September 2008 to September 2009, he was financially supported by China Scholarship Council (CSC) to work as a joint-training $\mathrm{PhD}$ student at the Nanyang Technological University (NTU), Singapore. In the light of his significant research at NTU, he received the Excellent Government-funded Scholars and Students Award in 2009. From August 2014 to August 2015, he worked as a Visiting Scholar at the University of Texas at San Antonio. He is currently a Full Professor with the School of Marine Electrical Engineering, DMU, China.

Dr. Wang received the Nomination Award of Liaoning Province Excellent Doctoral Dissertation, the DMU Excellent Doctoral Dissertation Award and the DMU Outstanding PhD Student Award in 2010, respectively. He also won the State Oceanic Administration Outstanding Young Scientists in Marine Science and Technology, the Liaoning Province Award for Technological Invention, the Liaoning BaiQianWan Talents, the Liaoning Excellent Talents, the Science and Technology Talents the Ministry of Transport of the P. R. China, the Youth Science and Technology Award of China Institute of Navigation, and the Dalian Leading Talents. He has authored over 200 book, journal, and refereed conference publications. His research interests include fuzzy neural systems, machine learning, nonlinear control, self-organizing fuzzy neural modeling and control, unmanned vehicles and autonomous control. He has been Leading Guest Editors of International Journal of Fuzzy Systems, International Journal of Advanced Robotics Systems, and Advances in Mechanical Engineering. He currently serves as Associate Editors of the Neurocomputing and the International Journal of Fuzzy Systems.

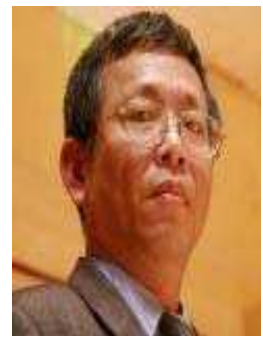

Shun-Feng Su (F'10) received the B.S. degree in electrical engineering from National Taiwan University, Taipei, Taiwan, in 1983 and the M.S. and $\mathrm{Ph} . \mathrm{D}$. degrees in electrical engineering from Purdue University, West Lafayette, IN, USA, in 1989 and 1991, respectively. He is currently a Chair Professor with the Department of Electrical Engineering, National Taiwan University of Science and Technology, Taipei. He has published more than 160 refereed journal and conference papers in the areas of robotics, intelligent control, fuzzy systems, neural networks, and nonderivative optimization. His current research interests include computational intelligence, machine learning, virtual reality simulation, intelligent transportation systems, smart home, robotics, and intelligent control.

Dr. Su is a Chinese Automatic Control Society (CACS) Fellow. He is very active in various international/domestic professional societies. $\mathrm{He}$ is the President of the Taiwan Fuzzy System Association and a Vice President of the International Fuzzy Systems Association. He currently serves on the Board of Governors of the CACS, the Taiwan Society of Robotics, and the Taiwan Association of System Science and Engineering. He has also served as a Program Chair, Program Co-chair, or PC member of various international and domestic conferences. He is currently an Associate Editor for the IEEE TRANSACTIONS ON CYBERNETICS and the IEEE TRANSACTIONS ON SYSTEMS, as well as an Area Editor of the International Journal of Fuzzy Systems.

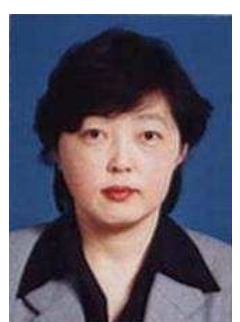

Min Han (M'95-A'03-SM'06) received the B.S. and M.S. degrees from the Department of Electrical Engineering, Dalian University of Technology, Dalian, China, in 1982 and 1993, respectively, and the M.S. and Ph.D. degrees from Kyushu University, Fukuoka, Japan, in 1996 and 1999, respectively. Since 2003, she has been a Professor with the Faculty of Electronic Information and Electrical Engineering, Dalian University of Technology. She has visited Washington University in St. Louis, St. Louis, MO, USA, in 2009. She has authored five books and over 300 articles in international journals and conference proceedings. Her current research interests include complex system modeling and forecasting method, time series analysis and forecasting, artificial intelligence method, neural networks and chaos and their applications in control and identification.

Dr. Han serves as a Deputy Director of the Chinese Society of Instrumentation Youth Work Committee and the Institute of Fuzzy Information Processing and Machine Intelligence of Dalian University of Technology, the Director of the Institute of Liaoning Province System Simulation, a Committee Member of the Chinese Society of Artificial Intelligence, a Consultant of Jiangsu Province Department of Science and Technology, and an Organizing Chair of ISNN2013, ICICIP 2014, and ICIST2016.

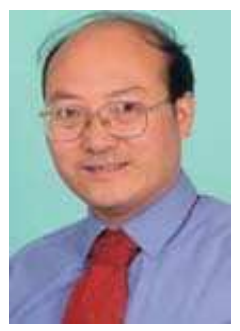

Wen-Hua Chen (SM'06-F'18) received the M.Sc. and Ph.D. degrees from Northeast University, Shenyang, China, in 1989 and 1991, respectively. From 1991 to 1996, he was a Lecturer and then Associate Professor with the Department of Automatic Control, Nanjing University of Aeronautics and Astronautics, Nanjing, China. From 1997 to 2000, he held a research position and then was a Lecturer of control engineering with the Centre for Systems and Control, University of Glasgow, Glasgow, U.K. In 2000, he moved to the Department of Aeronautical and Automotive Engineering, Loughborough University, Loughborough, U.K., as a Lecturer, where he was appointed as a Professor in 2012. His research interests include the development of advanced control strategies (nonlinear model predictive control, disturbance-observer-based control, etc.) and their applications in aerospace and automotive engineering. Currently, much of his work has also been involved with the development of unmanned autonomous intelligent systems.

Dr. Chen is a Fellow of The Institution of Engineering and Technology, U.K., and a Fellow of The Institution of Mechanical Engineers, U.K. 